\title{
Fermi and Non-Fermi Liquid Behavior of Quantum Impurity Models:* A Diagrammatic Pseudo-particle Approach
}

\author{
J. Kroha and P. Wölfle \\ Institut für Theorie der Kondensierten Materie, Universität Karlsruhe, 76128 Karlsruhe, Germany
}

\begin{abstract}
We review a systematic many-body method capable of describing Fermi liquid and Non-Fermi liquid behavior of quantum impurity models at low temperatures on the same footing. The crossover to the high temperature local moment regime is covered as well. The approach makes use of a pseudoparticle representation of the impurity Hilbert space in the limit of infinite Coulomb repulsion $U$ as well as for finite $U$. Approximations are derived from a generating Luttinger-Ward functional, in terms of renormalized perturbation theory in the hybridization $V$. Within a "conserving T-matrix approximation" (CTMA), including all two-particle vertex functions, an infinite series of leading infrared singular skeleton diagrams is included. The local constraint is strictly enforced. Applied to the $\mathrm{SU}(\mathrm{N}) \times \mathrm{SU}(\mathrm{M})$ multichannel Anderson model the method allows to recover the Fermi liquid behavior of the single channel case, as well as the non-Fermi liquid behavior in the multi-channel case. The results are compared with the "non-crossing approximation" (NCA) and with data obtained by the numerical renormalization group and the Bethe ansatz. The generalization of the method to the case of finite on-site repulsion $U$ is presented in a systematical way and solved on the level of a generalized NCA, fully symmetric with respect to all virtual excitations of the model.
\end{abstract}

\section{INTRODUCTION}

Over the past two decades the problem of correlated electrons on a lattice has emerged as a central theme of condensed matter theory. Since the mid seventies numerous metallic systems have been found in which the conduction electron system is believed to be dominated by a strong on-site Coulomb interaction. Examples are the heary fermion compounds 1 and the cuprate superconductors $\$$, but systems like the two-dimensional electron gas in quantum Hall devices 3 are governed by similar physics. Typical model Hamiltonians are the Hubbard model and the t-J model for the single-band situation, and the Anderson or Kondo lattice models in the multi-band case. In the event that the local Coulomb repulsion in these models exceeds the band width, conventional many-body theory, i.e. perturbation theory (including infinite resummations) in the Coulomb repulsion does not work anymore. The obvious alternative, perturbation expansion in terms of the kinetic energy meets with severe difficulty, mainly due to the infinite degeneracy of the ground state of a lattice model in the limit of zero hopping (a collection of independent atoms) and the non-canonical commutation relations of the field operators of the eigenstates in the atomic limit. It is therefore desirable to develop new methods which are capable of dealing with these problems.

To make it clear what is the principal obstacle for applying the well-established many-body techniques to this class of problems let us consider the Hubbard model with large on-site repulsion $U$, where each lattice site can either be empty (state $|0\rangle$ ), singly occupied $(|\uparrow\rangle,|\downarrow\rangle$ for an electron with spin projection $\uparrow, \downarrow)$ or doubly occu- pied $(|2\rangle)$. The dynamics of an electron will be very different according to whether it resides on a singly or doubly occupied site. For less than half band filling and large repulsive $U$ the doubly occupied states will be very costly in energy, and will contribute to the low energy physics only via virtual processes. This is the typical feature of strongly correlated electrons: the dynamics is constrained to a subspace of the total Hilbert space. The necessary projection onto this subspace is difficult to perform within conventional many-body theory and requires the development of new theoretical tools. This article deals with the method of auxiliary particles introduced to effect the projection in Hilbert space, while keeping most of the desirable features of renormalized perturbation theory.

The idea of auxiliary particles is simple: for each of the four states $|0\rangle,|\uparrow\rangle,|\downarrow\rangle,|2\rangle$ at a given lattice site (considering one orbital per site), a particle is introduced, which is created out of a vacuum state without any lattice site at all. The Fermi character of the electron requires that two of the auxiliary particles are fermions, e.g. the ones representing $|\uparrow\rangle$ and $|\downarrow\rangle$, and the remaining two are bosons. To ensure that a lattice site is in one of the four physical states, the number of auxiliary particles is constrained to be exactly one. Compared to alternative ways of effecting the projection in Hilbert space, the auxiliary particle method has the advantage of allowing one to use the machinery of quantum field theory, provided the constraint can be dealt with in a satisfactory way. As will be shown below, the constraint is closely related to a fictitious gauge symmetry in the Fock space of pseudo particles. The formulation in terms of renormalized perturbation theory in the kinetic energy presented in this paper

${ }^{*}$ Dedicated to Erwin Müller-Hartmann on the occasion of his 60th birthday 
allows to conserve the gauge symmetry in any chosen conserving approximation. Conserving approximations, on the other hand, are generated by functional derivation of a Luttinger-Ward functional. The Luttinger-Ward functional is chosen taking into account information on important physical processes as well as using expansion in a small parameter, e.g. the hybridization energy in units of the conduction electron band width. Auxiliary particle representations were pioneered by Abrikosovt, who first introduced a fermionic representation for local spins, and later by Barnes $\mathbf{E}$, who was the first to define and use the pseudo particle representation of a local impurity level we will be working with below.

Somewhat later these representations were applied to the N-fold degenerate Anderson-impurity models in conjunction with a mean-field approximation (MFA) of the slave boson fields 6 . As will be discussed below, the MFA, inspite of its initial success, suffers from severe problems: (1) it leads invariably to the Fermi liquid ground state, even for multi-channel models, in contradiction to the exact non-Fermi liquid behavior in those cases; (2) even for the single--channel case the MFA features a spurious first order transition separating the low temperature Kondo screened state from the high temperature local moment regime. On the technical side, the MFA violates the gauge symmetry mentioned above. The latter problem may be corrected by including the leading fluctuations around the saddle-point in $1 / N$ expansiond, whereas the former problems remain. We shall therefore avoid using the mean-field approximation which solves the problem of the spurious phase transition.

\section{SINGLE- AND MULTI-CHANNEL QUANTUM IMPURITY MODELS}

Quantum impurity models such as the Kondo and Anderson impurity models were discussed first in the context of magnetic impurities in metals $\mathrm{s}$. In these models an N-fold spin degenerate local impurity level (here called d-level) hybridizes with the conduction band. In the presence of a sufficiently strong Coulomb repulsion $U$ between any two electrons in the local impurity level and provided the level lies sufficiently far below the Fermi energy the local level will be singly occupied and a magnetic moment forms. The antiferromagnetic exchange interaction between the local moment and the local conduction electron spin density leads to the Kondo effect. The physics of the Kondo effect is not restricted to magnetic ions in metals. It depends only on the local level being degenerate (any two-level or multi-level system qualifies) and the local Coulomb interaction $U$ being sufficiently strong. The Kondo effect can therefore occur as well for quadrupole moments, the two-level systems in amorphous metals 9 or for the energy level system of quantum dots10. We will eqnsider the prototype Anderson impurity Hamiltonian 11

$$
H=H_{c}+H_{d}+H_{h y b}
$$

with the conduction electron Hamiltonian

$$
H_{c}=\sum_{\vec{k}, \sigma} \epsilon_{k} c_{\vec{k} \sigma} c_{\vec{k} \sigma} \quad ; \quad \sigma=1, \ldots N
$$

the local d-electron Hamiltonian

$$
H_{d}=E_{d} \sum_{\sigma} d_{\sigma}^{+} d_{\sigma}+U \sum_{\sigma<\sigma^{\prime}} n_{d \sigma} n_{d \sigma^{\prime}}
$$

and the hybridization term

$$
H_{h y b}=V \sum_{\vec{k} \sigma}\left(c_{\vec{k} \sigma}^{+} d_{\sigma}+h . c\right)
$$

In lowest order perturbation theory in $V$ the d-level at energy $E_{d}$ acquires a width $\Gamma=\pi V^{2} \mathcal{N}(0)$, with $\mathcal{N}(0)$ the conduction electron density of states at the Fermi level $\omega=0$. In the Kondo limit of the model, defined by $E_{d}<0,2 E_{d}+U>0, \Gamma /\left|E_{d}\right| \ll 1$ and $\Gamma /\left(2 E_{d}+U\right) \ll 1$, the low energy sector of the Anderson model may be mapped onto the Kondo mode12 featuring a local antiferromagnetic exchange interaction $J$ of the local spin of the single electron in the d-level with the local conduction electron spin, where $J=V^{2}\left[1 /\left|E_{d}\right|+1 /\left(2 E_{d}+U\right)\right]$. These models have been studied_extensively by means of Wilson'srenormalization group 13 , by the Bethe ansatz method 14.15 and by means of a phenomenological Fermi liquid theory 16 . In this way the following physical picture has emerged 8 : The model contains a dynamically generated low temperature scale, the Kondo temperature, which, for $U \rightarrow \infty$, is expressed in terms of the parameters of the Anderson Hamiltonian as

$$
T_{K}=D(N \Gamma / D)^{(M / N)} \mathrm{e}^{-\pi E_{d} / N \Gamma},
$$

with $\mathcal{N}(0)$ and $D=1 / \mathcal{N}(0)$ the density of states at the Fermi energy and the high energy band cutoff, respectively. $N, M$ are the degeneracy of the local level and the number of conduction electron channels (see below). In the intermediate temperature regime, $T \gtrsim T_{K}$, resonant spin flip scattering of electrons at the Fermi surface off the local degenerate level leads to logarithmic corrections to the magnetic susceptibility $\chi(T)$, the linear specific heat coefficient $\gamma(T)$ and the resistivity $\rho(T)$ and to a breakdown of perturbation theory at $T \simeq T_{K}$. In the single-channel case $(M=1)$, below $T_{K}$ a collective many-body spin singlet state develops in which the impurity spin is screened by the conduction electron spins as lower and lower energy scales are successively approached, leaving the system with a pure potential scattering center. The spin singlet formation is sketched in Fig. 1 a) and corresponds to a vanishing entropy at $T=0, S(0)=0$. It also leads to saturated behavior of physical quantities below $T_{K}$, like $\chi(T)=$ const, $\gamma(T)=$ $c(T) / T=$ const. and $\Delta \rho(T)=\rho(T)-\rho(0) \propto T^{2}$, i.e. to Fermi liquid behavior. 
a)
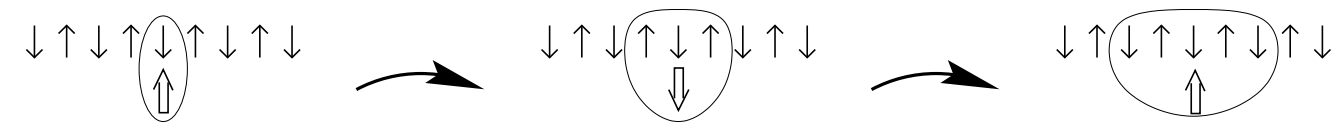

b)
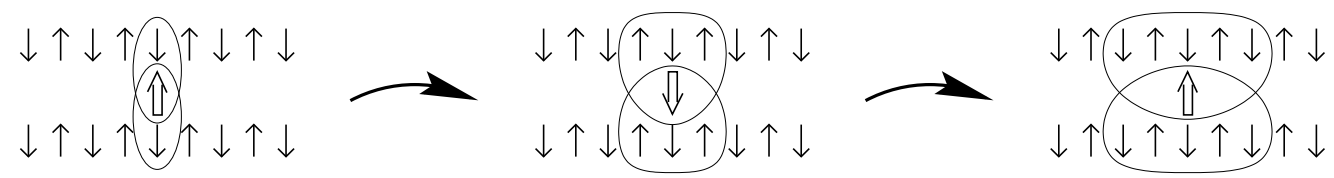

FIG. 1. Sketch of the renormalization group for a) the single-channel Kondo model (local moment compensation) and b) the two-channel Kondo model (local moment over-compensation). Small arrows denote conduction electron spins $1 / 2$, a heavy arrow a localized spin $1 / 2$. The curved arrows indicate successive renormalization steps.

Multichannel quantum impurity models are characterized by the completely symmetric coupling of $M$ degenerate conduction bands to the impurity. Let us assume for definiteness that the ground state of the impurity is a magnetic singlet, with $M$-fold orbital degeneracy, $\mid \Gamma \mu>$, where $\mu=1, \ldots M$, and the first excited state, obtained by adding one electron to the impurity, is magnetic and N-fold degenerate, but is an orbital singlet, $\left|\Gamma^{\prime} \sigma\right\rangle$, $\sigma=1, \ldots, N$. An $\mathrm{SU}(\mathrm{N}) \times \mathrm{SU}(\mathrm{M})$ symmetric Ander son model may then be defined in the limit $U \rightarrow \infty$ as

$$
H=H_{c \mu}=+E_{d} \sum_{\sigma}\left|\Gamma^{\prime} \sigma\right\rangle\left\langle\Gamma^{\prime} \sigma\right|+H_{h y b}
$$

where

$$
H_{c \mu}=\sum_{\vec{k} \sigma} \varepsilon_{\vec{k}} c_{\vec{k} \mu \sigma}^{\dagger} c_{\vec{k} \mu \sigma}
$$

describes the conduction electron continuum in orbital channel $\mu$ and

$$
H_{h y b}=V \sum_{\vec{k}, \sigma, \mu}\left(c_{\vec{k} \mu \sigma}^{+}|\Gamma \mu\rangle\left\langle\Gamma^{\prime} \sigma\right|+h . c .\right)
$$

and the effect of the Coulomb interaction is to restrict the average occupation to $n_{d}=\sum_{\sigma}\left|\Gamma^{\prime} \sigma\right\rangle\left\langle\Gamma^{\prime} \sigma\right| \leq 1$.

Like in the single channel case, in the multi-channel case, too, the conduction electrons of each channel separately screen the impurity moment by multiple spin scattering at temperatures below the Kondo scale $T_{K}$. However, in this case, the local moment is over-compensated, since the impurity spin can never form a singlet state with both conduction electron channels at the same time in this way, as can be seen in Fig. 1 b). As a consequence of this frustration, there is not a unique ground state, leading to a finite residual entropy 17 . 18 at $T=0$ of $S(0)=k_{B} \ln \sqrt{2}$ in the two-channel model. In particular, the precondition of FL theory of a 1:1 correspondence between interacting and non-interacting states is violated. As a conseqyence, characteristic singular temperature dependence $17, \mathrm{~L}$ of physical quantities persists for $T \lesssim T_{K}$ down to $T=0: \chi(T) \propto-\ln \left(T / T_{K}\right)$, $c(T) / T \propto-\ln \left(T / T_{K}\right)$ and $\rho(T)-\rho(0) \propto-\sqrt{T / T_{K}}$.

\section{PSEUDO-PARTICLE REPRESENTATION}

As discussed above, the dynamics of an electron occupying a local level of a quantum impurity will depend in an essential way on whether the level is singly occupied or multiply occupied, provided the Coulomb interaction $U$ between two electrons in the same level is large. It is therefore useful to divide the Hilbert space into sectors labeled by the occupation number of the quantum impurity. For each Fock state $\mid \alpha>$ of the impurity one may define a creation operator $a_{\alpha}^{+}$, which, when operating on a vacuum state $\mid$ vac $>$, creates the state $\mid \alpha>$ : $\left|\alpha>=a_{\alpha}^{+}\right|$vac $>$. For a usual impurity level with spin degeneracy $N=2$ one thus defines two bosons $b$ and $a$, and two fermions $f_{\sigma}$, $\sigma=\uparrow, \downarrow$, creating the empty and doubly occupied states $\left|0>=b^{+}\right| \operatorname{vac}>,\left|2>=a^{+}\right| \operatorname{vac}>$, and the singly occupied states $\left|\sigma>=f_{\sigma}^{+}\right| \operatorname{vac}>$. The creation of an electron in the empty level, effected by a fermion creation operator $d_{\sigma}^{+}$, according to $\left|\sigma>=d_{\sigma}^{+}\right| 0>$ or $\left.\left|2>=d_{\uparrow}^{+}\right| \downarrow\right\rangle=-d_{\downarrow}^{+}|\uparrow\rangle$, is described in terms of the pseudo-particle operators as

$$
d_{\sigma}^{+}=f_{\sigma}^{+} b+\eta_{\sigma} a^{+} f_{-\sigma}
$$

where the factor $\eta_{\sigma}= \pm 1$ for $\sigma=\uparrow, \downarrow$ accounts for the antisymmetry of the doubly occupied state. By the introduction of the pseudo-particles the Fock space has been vastly extended. The physical sector of this artificial Fock space is defined by the requirement that the impurity should be occupied by exactly one pseudo-particle at any time (corresponding to a definite state), as expressed by the constraint 


$$
\sum_{\sigma} f_{\sigma}^{+} f_{\sigma}+b^{+} b+a^{+} a=1
$$

In the following we will largely consider the case of infinite Coulomb repulsion $U$, implying that double (multiple) occupancy of the impurity is not possible. The operators $a^{+}$, $a$ will therefore not appear in this case. In the multi-channel $\mathrm{SU}(\mathrm{N}) \times \mathrm{SU}(\mathrm{M})$ Anderson model discussed in the last section, we will, however, need to introduce $M$ boson operators $b_{\mu}^{+}, \mu=1, \ldots M$ creating the $M$ ground states $|\Gamma \mu\rangle$, and $N$ fermion operators $f_{\sigma}^{+}, \sigma=1, \ldots N$. In terms of these operators the Hamiltonian of the $\mathrm{SU}(\mathrm{N}) \times \mathrm{SU}(\mathrm{M})$ Anderson model takes the form

$$
\begin{aligned}
H & =\sum_{\vec{k}, \sigma, \mu} \varepsilon_{\vec{k}} c_{\vec{k} \mu \sigma}^{\dagger} c_{\vec{k} \mu \sigma}+E_{d} \sum_{\sigma} f_{\sigma}^{\dagger} f_{\sigma} \\
& +V \sum_{\vec{k}, \sigma, \mu}\left(c_{\vec{k} \mu \sigma}^{\dagger} b_{\vec{\mu}}^{\dagger} f_{\sigma}+\text { h.c. }\right)
\end{aligned}
$$

In order for $H$ to be $\mathrm{SU}(\mathrm{M})$ invariant, the slave boson multiplet $b_{\bar{\mu}}$ transforms according to the conjugate representation of the $\mathrm{SU}(\mathrm{M})$. In addition, the operator constraint

$$
Q \equiv \sum_{\sigma} f_{\sigma}^{\dagger} f_{\sigma}+\sum_{\mu} b_{\bar{\mu}}^{\dagger} b_{\bar{\mu}}=1
$$

has to be satisfied at all times. One might interpret the constraint as a statement of charge quantization, with the integer $Q$ the conserved, quantized charge. Similar to quantum field theories with conserved charges, the charge conservation is intimately related to the existence of a local gauge symmetry. Indeed, the system defined by the Hamiltonian Eq. (11) is invariant under simultaneous local $U(1)$ gauge transformations $f_{\sigma} \rightarrow f_{\sigma} \mathrm{e}^{i \phi(\tau)}$, $b_{\bar{\mu}} \rightarrow b_{\bar{\mu}} \mathrm{e}^{i \phi(\tau)}$, with $\phi(\tau)$ an arbitrary time dependent phase.

\section{A. Exact projection onto the physical Hilbert space}

While the gauge symmetry guarantees the conservation of the quantized charge $Q$, it does not single out any particular $Q$, such as $Q=1$. In order to effect the projection onto the sector of Fock space with $Q \overline{\overline{7}} 1$, one may use a procedure first proposed by Abrikosovt: Consider first the grand-canonical ensemble with respect to $Q$, defined by the statistical operator

$$
\hat{\rho}_{G}=\frac{1}{Z_{G}} \mathrm{e}^{-\beta(H+\lambda Q)},
$$

where $Z_{G}=\operatorname{tr}[\exp \{-\beta(H+\lambda Q)\}]$ is the grand-canonical partition function with respect to $Q,-\lambda$ is the associated chemical potential, and the trace extends over the complete Fock space, including summation over $Q$. The expectation value of an observable $\hat{A}$ in the grand-canonical ensemble is given by

$$
\langle\hat{A}\rangle_{G}=\operatorname{tr}\left[\hat{\rho}_{G} \hat{A}\right]
$$

The physical expectation value of $\hat{A},\langle\hat{A}\rangle$, is to be evaluated in the canonical ensemble where $Q=1$. It can be calculated from the grand-canonical ensemble by differentiating with respect to the fugacity $\zeta=\mathrm{e}^{-\beta \lambda}$ and taking $\lambda$ to infinity 6 ,

$$
\langle\hat{A}\rangle=\lim _{\lambda \rightarrow \infty} \frac{\frac{\partial}{\partial \zeta} \operatorname{tr}\left[\hat{A} e^{-\beta(H+\lambda Q)}\right]}{\frac{\partial}{\partial \zeta} \operatorname{tr}\left[e^{-\beta(H+\lambda Q)}\right]}=\lim _{\lambda \rightarrow \infty} \frac{\langle Q \hat{A}\rangle_{G}}{\langle Q\rangle_{G}} .
$$

Projecting operators acting on the impurity states:We list two important results, which follow straightforwardly from Eq. (15): First, the canonical partition function in the subspace $Q=1$ is

$$
\begin{aligned}
Z_{C} & =\lim _{\lambda \rightarrow \infty} \operatorname{tr}\left[Q e^{-\beta(H+\lambda(Q-1))}\right] \\
& =\lim _{\lambda \rightarrow \infty}\left(e^{\beta \lambda}\langle Q\rangle_{G}(\lambda)\right) Z_{Q=0},
\end{aligned}
$$

where the subscripts $G$ and $C$ denote the grand-canonical and the canonical $(Q=1)$ expectation value, respectively. Second, the canonical $Q=1$ expectation value of any operator $\hat{A}$ which has a zero expectation value in the $Q=0$ subspace, $\hat{A}|Q=0\rangle=0$, is given by,

$$
\langle\hat{A}\rangle_{C}=\lim _{\lambda \rightarrow \infty} \frac{\langle\hat{A}\rangle_{G}(\lambda)}{\langle Q\rangle_{G}(\lambda)}
$$

Note that $\hat{A}|Q=0\rangle=0$ holds true for any physically observable operator acting on the impurity. Examples are the physical electron operator $d_{\mu \sigma}^{\dagger}=f_{\sigma}^{\dagger} b_{\bar{\mu}}$ or the local spin operator $\vec{S}=\sum_{\sigma \sigma^{\prime}} \frac{1}{2} f_{\sigma}^{\dagger} \vec{\tau}_{\sigma \sigma^{\prime}} f_{\sigma^{\prime}}$. In this case the operator $Q$ appearing in the numerator of Eq. (15) is not necessary to project away the $Q=0$ sector. In particular, the constrained $d$-electron Green's function is given in terms of the grand-canonical one $\left(G_{d}(\omega, T, \lambda)\right)$ as

$$
G_{d}(\omega)=\lim _{\lambda \rightarrow \infty} \frac{G_{d}(\omega, T, \lambda)}{\langle Q\rangle_{G}(\lambda)}
$$

In the enlarged Hilbert space $(Q=0,1,2, \ldots) G_{d}(\omega, T, \lambda)$ may be expressed in terms of the grand-canonical pseudo-fermion and slave boson Green's functions using Wick's theorem. These auxiliary particle Green's functions, which constitute the basic building blocks of the theory, are defined in imaginary time representation as

$$
\begin{aligned}
\mathcal{G}_{f \sigma}\left(\tau_{1}-\tau_{2}\right) & =-\left\langle\hat{T}\left\{f_{\sigma}\left(\tau_{1}\right) f_{\sigma}^{\dagger}\left(\tau_{2}\right)\right\}\right\rangle_{G} \\
\mathcal{G}_{b \bar{\mu}}\left(\tau_{1}-\tau_{2}\right) & =-\left\langle\hat{T}\left\{b_{\bar{\mu}}\left(\tau_{1}\right) b_{\bar{\mu}}^{\dagger}\left(\tau_{2}\right)\right\}\right\rangle_{G}
\end{aligned}
$$

where $\hat{T}$ is the time ordering operator. The Fourier transforms of $\mathcal{G}_{f, b}$ may be expressed in terms of the exact selfenergies $\Sigma_{f, b}$ as

$$
\mathcal{G}_{f, b}\left(i \omega_{n}\right)=\left\{\left[\mathcal{G}_{f, b}^{0}\left(i \omega_{n}\right)\right]^{-1}-\Sigma_{f, b}\left(i \omega_{n}\right)\right\}^{-1}
$$


where

$$
\begin{aligned}
\mathcal{G}_{f \sigma}^{0}\left(i \omega_{n}\right) & =\left(i \omega_{n}-E_{d}-\lambda\right)^{-1} \\
\mathcal{G}_{b \bar{\mu}}^{0}\left(i \omega_{n}\right) & =\left(i \omega_{n}-\lambda\right)^{-1}
\end{aligned}
$$

Since, as a consequence of the projection procedure $\lambda \rightarrow$ $\infty$, the energy eigenvalues of $H+\lambda Q$ scale to infinity as $\lambda Q$, it is useful to shift the zero of the auxiliary particle frequency scale by $\lambda$ (in the $Q=1$ sector) and to define the "projected" Green's functions as

$$
G_{f, b}(\omega)=\lim _{\lambda \rightarrow \infty} \mathcal{G}_{f, b}(\omega+\lambda) .
$$

Note that this does not affect the energy scale of physical quantities (like the local $d$ electron Green's function), which is the difference between the the pseudo-fermion and the slave-boson energy.

Canonical expectation values of conduction electron operators:- The canonical (i.e. projected onto the $Q=1$ subspace), local conduction electron Green function is given as

$$
G_{c \mu \sigma}\left(i \omega_{n}\right)=\left\{\left[G_{c \mu \sigma}^{0}\left(i \omega_{n}\right)\right]^{-1}-\Sigma_{c \mu \sigma}\left(i \omega_{n}\right)\right\}^{-1}
$$

with

$$
G_{c \mu \sigma}^{0}\left(i \omega_{n}\right)=\sum_{\vec{k}} G_{c \mu \sigma}^{0}\left(\vec{k}, i \omega_{n}\right)=\sum_{\vec{k}}\left(i \omega_{n}+\mu_{c}-\epsilon_{\vec{k}}\right)^{-1},
$$

where $\mu_{c}$ is the chemical potential of the conduction electrons. The canonical, local $c$-electron self-energy, $\Sigma_{c \mu \sigma}\left(i \omega_{n}\right)$, cannot be obtained from the grand-canonical one by simply taking the limit $\lambda \rightarrow \infty$, since the $c$ electron density has a non-vanishing expectation value in the $Q=0$ subspace. However, it follows immediately from the Anderson Hamiltonian, Eqs. (6)-(8), that the exact, canonical conduction electron t-matrix $t_{\sigma \mu}(i \omega)$, defined by $G_{c}=G_{c}^{0}\left[1+t G_{c}^{0}\right]$, is proportional to the full, projected $d$-electron Green's function, $t_{\mu \sigma}(i \omega)=$ $|V|^{2} G_{d \mu \sigma}$. Thus, we have as an exact relation,

$$
G_{c \mu \sigma}\left(i \omega_{n}\right)=G_{c \mu \sigma}^{0}\left(i \omega_{n}\right)\left[1+|V|^{2} G_{d \mu \sigma}\left(i \omega_{n}\right) G_{c \mu \sigma}^{0}\left(i \omega_{n}\right)\right],
$$

and by comparison with Eq. (25) we obtain the local conduction electron self-energy respecting the constrained dynamics in the impurity orbital,

$$
\Sigma_{c \mu \sigma}\left(i \omega_{n}\right)=\frac{|V|^{2} G_{d \mu \sigma}\left(i \omega_{n}\right)}{1+|V|^{2} G_{c \mu \sigma}^{0}\left(i \omega_{n}\right) G_{d \mu \sigma}\left(i \omega_{n}\right)} .
$$

Using phenomenological Fermi liquid theory 16 and also by means of perturbation theory to infinite order in the on-site repulsion $U$ el it has been shown for the Fermi liquid case $M=1$ of the symmetric Anderson model $\left(2 E_{d}=\right.$ $-U)$ that the exact $d$-electron propagator $G_{d \sigma}(\omega)$ and the $d$-electron self-energy $\Sigma_{d \sigma}(\omega) \equiv \omega-G_{d \sigma}(\omega)^{-1}$ obey the following local Fermi liquid relations in the limit $\omega \rightarrow 0-i 0, T \rightarrow 0$,

$$
\begin{aligned}
& \text { Luttinger theorem: } \int d \omega f(\omega) \frac{\partial \Sigma_{d \sigma}(\omega)}{\partial \omega} G_{d \sigma}(\omega)=0 \\
& \text { Friedel-Langreth: } \frac{1}{\pi} \operatorname{Im} G_{d \sigma}(\omega)=\frac{1}{\Gamma} \sin ^{2}\left(\frac{\pi n_{d}}{N}\right)-c\left[\left(\frac{\omega}{T_{K}}\right)^{2}+\left(\frac{\pi T}{T_{K}}\right)^{2}\right] \\
& \operatorname{Im} \Sigma_{d \sigma}(\omega)=\frac{\Gamma}{\sin ^{2}\left(\pi n_{d} / N\right)}+c\left(\frac{\Gamma}{\sin ^{2}\left(\pi n_{d} / N\right)}\right)^{2}\left[\left(\frac{\omega}{T_{K}}\right)^{2}+\left(\frac{\pi T}{T_{K}}\right)^{2}\right],
\end{aligned}
$$

where $c$ is a constant of $O(1)$. Combining Eqs. 28), (30) it follows that (for $M=1$, away from particle hole symmetry) $\Sigma_{c \sigma}$ exhibits (in an exact theory) local Fermi liquid behavior as well, $\operatorname{Im} \Sigma_{c \sigma}(\omega-i 0, T=0)=$ $a+b\left(\omega / T_{K}\right)^{2}$ for $\omega \rightarrow 0$. Note that this quantity is different from the grand-canonical conduction electron self-energy and has a finite imaginary part at the Fermi level.

The momentum dependent conduction electron Green's function in the presence of a single impurity is given in terms of the canonical $d$-electron propagator as

$$
G_{c \mu \sigma}\left(\vec{k}, \vec{k}^{\prime} ; i \omega_{n}\right) G_{c \mu \sigma}^{0}\left(\vec{k}, i \omega_{n}\right)\left[\delta_{\vec{k}, \vec{k}^{\prime}}+|V|^{2} G_{d}\left(i \omega_{n}\right) G_{c \mu \sigma}^{0}\left(\vec{k}^{\prime}, i \omega_{n}\right)\right] .
$$

The latter expression is the starting point for treating a random system of many Anderson impurities 21 . 


\section{B. Analytical properties and infrared behavior}

The Green's functions $G_{f, b, c}$ have the following spectral representations

$$
G_{f, b, c}\left(i \omega_{n}\right)=\int_{-\infty}^{\infty} d \omega^{\prime} \frac{A_{f, b, c}\left(\omega^{\prime}\right)}{i \omega_{n}-\omega^{\prime}}
$$

with the normalization of the spectral functions $A_{f, b, c}$

$$
\int_{-\infty}^{\infty} d \omega A_{f, b, c}(\omega)=1 .
$$

Taking the limit $\lambda \rightarrow \infty$ has important consequences on the analytical structure of the auxiliary particle Green's functions:
(1) - It follows directly from the definitions Eqs. (24), (20), using Eqs. (13), (14), that the traces appearing in the canonical functions $G_{f}$ are taken purely over the $Q=0$ sector of Fock space 22 . Thus, the backward-intime $\left(\tau_{1}<\tau_{2}\right)$ or hole-like contribution to the auxiliary propagators in Eq. (20) vanishes after projection, and we have

$$
\begin{aligned}
G_{f \sigma}\left(\tau_{1}-\tau_{2}\right) & =-\Theta\left(\tau_{1}-\tau_{2}\right) \lim _{\lambda \rightarrow \infty}\left\langle f_{\sigma}\left(\tau_{1}\right) f_{\sigma}^{\dagger}\left(\tau_{2}\right)\right\rangle_{G} \\
G_{b \bar{\mu}}\left(\tau_{1}-\tau_{2}\right) & =-\Theta\left(\tau_{1}-\tau_{2}\right) \lim _{\lambda \rightarrow \infty}\left\langle b_{\bar{\mu}}\left(\tau_{1}\right) b_{\bar{\mu}}^{\dagger}\left(\tau_{2}\right)\right\rangle_{G} .
\end{aligned}
$$

Consequently, their spectral functions $A_{f, b}$ have the Lehmann representation

$$
\begin{aligned}
& A_{f \sigma}(\omega)=\sum_{m, n \geq 0} \mathrm{e}^{-\beta E_{m}^{0}}\left|\left\langle 1, n\left|f_{\sigma}^{\dagger}\right| 0, m\right\rangle\right|^{2} \delta\left(\omega-\left(E_{n}^{1}-E_{m}^{0}\right)\right) \\
& A_{b \bar{\mu}}(\omega)=\sum_{m, n \geq 0} \mathrm{e}^{-\beta E_{m}^{0}}\left|\left\langle 1, n\left|b_{\bar{\mu}}^{\dagger}\right| 0, m\right\rangle\right|^{2} \delta\left(\omega-\left(E_{n}^{1}-E_{m}^{0}\right)\right),
\end{aligned}
$$

where $E_{n}^{Q}$ are the energy eigenvalues $\left(E_{0}^{0} \leq E_{n}^{Q}\right.$ is the ground state energy) and $|Q, n\rangle$ the many-body eigenstates of $H$ in the sector $Q$ of Fock space. At zero temperature, $A_{f}$ reduces to $A_{f \sigma}(\omega)=\sum_{n \geq 0}\left|\left\langle 1, n\left|f_{\sigma}^{\dagger}\right| 0,0\right\rangle\right|^{2}$ $\delta\left(\omega-\left(E_{n}^{1}-E_{0}^{0}\right)\right)$ and similar for $A_{b}$. It is seen that the $A_{f, b}$ have threshold behavior at $\omega=E_{0} \equiv E_{0}^{1}-E_{0}^{0}$, with $A_{f, b}(\omega) \equiv 0$ for $\omega<E_{0}, T=0$. The vanishing imaginary part at frequencies $\omega<0$ may be shown to be a general property of all quantities involving slave particle operators, e.g. also of auxiliary particle self-energies and vertex functions.

(2) - As will be seen in section V B (Eq. (54)), phys- ical expectation values not only involve the particle-like auxiliary propagators Eqs. (35), (36) but also hole-like contributions. It is, therefore, useful to define the "antifermion" and "anti-boson" propagators (in imaginary time representation)

$$
\begin{aligned}
G_{f \sigma}^{-}\left(\tau_{1}-\tau_{2}\right) & =-\Theta\left(\tau_{2}-\tau_{1}\right) \lim _{\lambda \rightarrow \infty}\left\langle f_{\sigma}^{\dagger}\left(\tau_{2}\right) f_{\sigma}\left(\tau_{1}\right)\right\rangle_{G} \\
G_{b \bar{\mu}}^{-}\left(\tau_{1}-\tau_{2}\right) & =-\Theta\left(\tau_{2}-\tau_{1}\right) \lim _{\lambda \rightarrow \infty}\left\langle b_{\bar{\mu}}^{\dagger}\left(\tau_{2}\right) b_{\bar{\mu}}\left(\tau_{1}\right)\right\rangle_{G},
\end{aligned}
$$

whose spectral functions have the Lehmann representations

$$
\begin{aligned}
& A_{f \sigma}^{-}(\omega)=\sum_{m, n \geq 0} \mathrm{e}^{-\beta E_{m}^{1}}\left|\left\langle 0, n\left|f_{\sigma}\right| 1, m\right\rangle\right|^{2} \delta\left(\omega-\left(E_{n}^{0}-E_{m}^{1}\right)\right) \\
& A_{b \bar{\mu}}^{-}(\omega)=\sum_{m, n \geq 0} \mathrm{e}^{-\beta E_{m}^{1}}\left|\left\langle 0, n\left|b_{\bar{\mu}}\right| 1, m\right\rangle\right|^{2} \delta\left(\omega-\left(E_{n}^{0}-E_{m}^{1}\right)\right) .
\end{aligned}
$$

$E_{0}^{1}$ is the ground state energy in the $Q=1$ sector. The expressions (37), (38) and (41), (42) immediately imply a relation between $A_{f, b}$ and $A_{f, b}^{-}$,

$$
A_{f, b}^{-}(\omega)=\mathrm{e}^{-\beta \omega} A_{f, b}(\omega)
$$

(3) — The property of only forward-in-time propagation (Eqs. (35), (36)) means that the auxiliary particle propagators $G_{f, b}$ are formally identical to the core hole profagators of the well-known X-ray threshold problem23 25. Thus, the knowledge of the infrared behavior of the latter may be directly applied to the former. In particular, the spectral functions are found (see below) to diverge at the threshold $E_{0}$ in a power law fashion (infrared singularity)

$$
A_{f, b}(\omega) \sim\left|\omega-E_{0}\right|^{-\alpha_{f, b}} \theta\left(\omega-E_{0}\right)
$$

due to a diverging number of particle-hole excitation processes in the conduction electron sea as $\omega \rightarrow E_{o}$. For the single channel case $(M=1)$, i.e. the usual Kondo or mixed valence problem, the exponents $\alpha_{f}$ and $\alpha_{b}$ can be found analytically from the following chain of arguments: Anticipating that in this case the impurity spin is completely screened by the conduction electrons at temperature $T=0$, leaving a pure-potential scattering center, the ground state $|1,0\rangle$ is a Slater determinant of one-particle scattering states, characterized by scattering 
phase shifts $\eta_{\sigma}$ in the s-wave channel (assuming for simplicity a momentum independent hybridization matrix element $V$ ). To calculate the fermion spectral function $A_{f \sigma}(\omega)$ at $T=0$ from Eq. (37), one needs to evaluate $\left\langle 1, n\left|f_{\sigma}^{\dagger}\right| 0,0\right\rangle$, which is just the overlap of two slater determinants, an eigenstate of the fully interacting Kondo system, $|1, n\rangle$, on the one hand, and the ground state of the conduction electron system in the absence of the impurity combined with the decoupled impurity level occupied by an electron with spin $\sigma, f_{\sigma}^{\dagger}|0,0\rangle$, on the other hand. As shown by Anderson23, the overlap of the two ground state slater determinants, $\left\langle 1,0\left|f_{\sigma}^{\dagger}\right| 0,0\right\rangle$, tends to zero in the thermodynamic limit (orthogonality catastrophe). Analogous relations hold for the boson spectral function $A_{b}(\omega)$. As a result, the exponential relaxation into the interacting ground state for long times is inhibited, leading to the infrared power law divergence of the spectral functions, Eq. (44).

The X-ray threshold exponents can be expressed in terms of the scattering phase shifts at the Fermi level by the exact relation 26

$$
\alpha_{f, b}=1-\sum_{\sigma^{\prime}}\left(\frac{\eta_{f, b \sigma^{\prime}}}{\pi}\right)^{2} .
$$

Here the $\eta_{f \sigma^{\prime}}\left(\eta_{b \sigma^{\prime}}\right)$ are the scattering phase shifts of the single-particle wave functions in channel $\sigma^{\prime}$ of the fully interacting ground state $|1,0\rangle$, relative to the wave functions of the free state $f_{\sigma}^{\dagger}|0,0\rangle\left(b^{\dagger}|0,0\rangle\right)$. Via the Friedel sum rule, the scattering phase shifts are, in turn, related to the change $\Delta n_{c \sigma^{\prime}}$ of the average number of conduction electrons per scattering channel $\sigma^{\prime}$ due to the presence of the impurity: $\eta_{f, b \sigma^{\prime}}=\pi \Delta n_{c \sigma^{\prime}}$. Obviously, $\Delta n_{c \sigma^{\prime}}$ is equal and opposite in sign to the difference of the average impurity occupation numbers of the states $|1,0\rangle$ and $f_{\sigma}^{\dagger}|0,0\rangle\left(b^{\dagger}|0,0\rangle\right)$. Thus, in the pseudofermion propagator $G_{f \sigma}$ we have the phase shifts,

$$
\eta_{f \sigma^{\prime}}=-\pi\left(\frac{n_{d}}{N}-\delta_{\sigma \sigma^{\prime}}\right)
$$

and in the slave boson propagator $G_{b}$,

$$
\eta_{b}=-\pi \frac{n_{d}}{N}
$$

where $n_{d}$ denotes the total occupation number of the impurity level in the interacting ground state. (The term $\delta_{\sigma \sigma^{\prime}}$ in $\eta_{f \sigma^{\prime}}$ appears because $f_{\sigma}^{\dagger}|0,0\rangle$ has impurity occupation number 1 in the channel $\sigma$.) For example, in the Kondo limit $n_{d} \rightarrow 1$ and for a spin $1 / 2$ impurity $(N=2)$ this leads to resonance scattering, $\eta_{f, b \sigma^{\prime}}=\pi / 2$. As a result, one find 27 for the threshold exponents

$$
\begin{aligned}
\alpha_{f} & =\frac{2 n_{d}-n_{d}^{2}}{N} \\
\alpha_{b} & =1-\frac{n_{d}^{2}}{N}
\end{aligned}
$$

These results have been found independently frem Wilson's numerical renormalization group approach 28.29 and using the Bethe ansatz solution and boundary conformal field theory 30 . It is interesting to note that (i) the exponents depend on the level occupancy $n_{d}$ (in the Kondo limit $n_{d} \rightarrow 1, \alpha_{f}=1 / N$ and $\alpha_{b}=1-1 / N$, whereas in the opposite, empty orbital, limit $n_{d} \rightarrow 0$, $\alpha_{f} \rightarrow 0$ and $\alpha_{b} \rightarrow 1$ ) (ii) the sum of the exponents $\alpha_{f}+\alpha_{b}=1+2 \frac{n_{d}\left(1-n_{d}\right)}{N} \geq 1$.

We stress that the above derivation of the infrared exponents $\alpha_{f, b}$ holds true only if the impurity complex acts as a pure potential scattering center at $T=0$. This is equivalent to the statement that the conduction electrons behave locally, i.e. at the impurity site, like a Fermi liquid. Conversely, in the multi-channel (non-FL) case, $N \geq 2, M \geq N$, the exponents have been found from a conformal field theory solution 19 of the problem in the Kondo limit to be

$$
\begin{gathered}
\alpha_{f}=M /(M+N) \\
\alpha_{b}=N /(M+N)
\end{gathered}
$$

which differ from the FL values. Thus, one may infer from the values of $\alpha_{f, b}$ as a function of $n_{d}$, whether or not the system is in a local Fermi liquid state.

\section{MEAN FIELD APPROACH AND $1 / N$ EXPANSION AT $U \rightarrow \infty$}

For physical situations of interest, the $s-d$ hybridization of the Anderson model (11) is much smaller than the conduction band width, $\mathcal{N}(0) V \ll 1$, where $\mathcal{N}(0)=1 / D$ is the local conduction electron density of states at the Fermi level. This suggests a perturbation expansion in $\mathcal{N}(0) V$. A straightforward expansion in terms of bare Green's functions is not adequate, as it would not allow to capture the physics of the Kondo screened state, or else the infrared divergencies of the auxiliary particle spectral functions discussed in the last section. In the framework of the slave boson representation, two types of nonperturbative approaches have been developed. The first one is mean field theory for both the slave boson amplitude $\langle b\rangle$ and the constraint $(\langle Q\rangle=1$ rather than $Q=1)$. The second one is resummation of the perturbation theory to infinite order.

\section{A. Slave boson mean field theory}

Slave boson mean field theory is based on the assumption that the slave bosons condense at low temperatures such that $\left\langle b_{\bar{\mu}}\right\rangle \neq 0$ Replacing the operator $b_{\bar{\mu}}$ in $H+\lambda Q$ by $\left\langle b_{\bar{\mu}}\right\rangle$ (see Ref.31), where $\lambda$ is a Lagrange multiplier to be adjusted such that $\langle Q\rangle=1$, one arrives at a resonance level model for the pseudofermions. The position of the resonance, $E_{d}+\lambda$, is found to be given by the Kondo temperature $T_{K}$, and is thus close to the Fermi 
energy. The resonance generates the low energy scale $T_{K}$, and leads to local Fermi liquid behavior. While this is qualitatively correct in the single-channel case, it is in blatant disagreement with the exactly known behavior in the multi-channel case. The mean field theory can be shown to be exact for $M=1$ in the limit $N \rightarrow \infty$ for a model in which the constraint is softened to be $Q=N / 2$. However, for finite $N$ the breaking of the local gauge symmetry, which would be implied by the condensation of the slave boson field, is forbidden by Elitzur's theorem 32 . It is known that for finite $N$ the fluctuations in the phase of the complex expectation value $\left\langle b_{\bar{\mu}}\right\rangle$ are divergent and lead to the suppression of $\left\langle b_{\bar{\mu}}\right\rangle$ to zero (see also 33 35). This is true in the cartesian gauge, whereas in the radial gauge the phase fluctuations may be shown to cancel at least in lowest order. It has not been possible to connect the mean field solution, an apparently reasonable description at low temperatures and for $M=1$, to the high temperature behavior $\left(T \gg T_{K}\right)$, dominated by logarithmic temperature dependence, in a continuous way 31 . Therefore, it seems that the slave boson mean field solution does not offer a good starting point even for only a qualitatively correct description of quantum impurity models.

\section{B. 1/N expansion vs. self-consistent formulation}

The critical judgement of mean field theory is corroborated by the results of a straightforward $1 / N$-expansion in the single channel case, keeping the exact constraint, and npt allowing for a finite bose field expectation value 36 . Within this scheme the exact behavior of the thermodynamic quantities (known from the Bethe ansatz solution) at low temperatures as well as high temperatures is recovered to the considered order in $1 / N$. Also, the exact auxiliary particle exponents $\alpha_{f, b}$ are reproduced in order $1 / N$, using a plausible exponentiation scheme 37 .

In addition, dynamical quantities like the d-electron spectral function and transport coefficients can be calculated exactly to a desired order in $1 / N$ within this approach. However, as clear-cut and economical this method may be, it does have serious limitations. For once, the $1 / N$ expansion is not uniformly convergent as a function of temperature 38 . Rather, the expansions at low temperature and at high temperature have to be done around two different saddle-points (the limits $N \rightarrow \infty)$. It is not known how to match these expansions in the crossover region $T \sim T_{K}$ in a systematic way. Secondly, the experimentally most relevant case of $N=2$ or somewhat larger is not accessible in $1 / N$ expansion. Thirdly, non-Fermi liquid behavior, being necessarily non-perturbative in $1 / N$, cannot be dealt with in a controlled way on the basis of a $1 / N$-expansion. To access these latter two regimes, a new approach nonperturbative in $1 / N$ is necessary.

\section{CONSERVING APPROXIMATIONS: GAUGE INVARIANT SELF-CONSISTENT PERTURBATION THEORY IN THE HYBRIDIZATION}

We conjecture that this new approach is gauge invariant many-body theory of pseudofermions and slave bosons. As long as gauge symmetry violating objects such as Bose field expectation values or fermion pair correlation functions do not appear in the theory, gauge invariance of physical quantities can be guaranteed in suitably chosen approximations by the proper match of pseudofermion and slave boson properties, without introducing an additional gauge field. This requires the use of conserving approximations 39 , derived from a LuttingerWard functional $\Phi$.

\section{A. Generating functional}

$\Phi$ consists of all vacuum skeleton diagrams built out of fully renormalized Green's functions $G_{b, f, c}$ and the bare vertex $V$. The self-energies $\Sigma_{b, f, c}$ are obtained by taking the functional derivative of $\Phi$ with respect to the corresponding Green's function (cutting the Green's function line in each diagram in all possible ways),

$$
\Sigma_{b, f, c}=\delta \Phi / \delta G_{b, f, c}
$$

Irreducible vertex functions, figuring as integral kernels in two-particle Bethe-Salpeter equations, are generated by second order derivatives of $\Phi$.

The choice of diagrams for $\Phi$ defines a given approximation. It should be dictated by the dominant physical processes and by expansion in a small parameter, if available. Even in the presence of a small parameter (in this case $\mathcal{N}_{0} V$ ), straightforward low order renormalized perturbation theory may not give an even qualitatively correct result, if singular vertex functions appear. It is therefore necessary to check whether vertex functions become singular at the level where approximate single particle Green's functions are used to calculate the integral kernels of the respective vertex functions. Should this be the case, the vertex functions have to be included into the approximation in a gauge invariant way. As we shall see, in the present case it is required to take all two-particle vertex functions into consideration. Vertex functions involving three or more interacting particles will be omitted in the hope that the corresponding phase space is small so that they will contribute less even if they are singular. This is the first fundamental approximation. The second one is that we will approximate the irreducible kernels in the Bethe-Salpeter equations for the vertex functions by the lowest order diagram in $\mathcal{N}_{0} V$. 


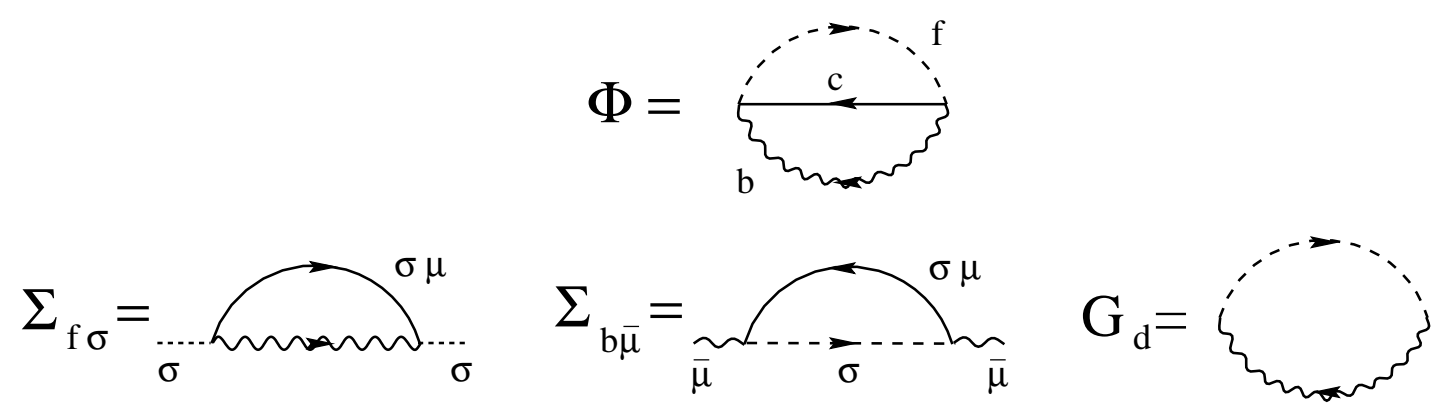

FIG. 2. Diagrammatic representation of the generating functional $\Phi$ of the NCA. Also shown are the pseudoparticle self-energies and the local electron Green's function derived from $\Phi$, Eqs. (19)-(21). Throughout this article, dashed, wavy and solid lines represent fermion, boson, and conduction electron lines, respectively. In the diagram for $\Sigma_{f \sigma}$ the spin labels are shown explicitly to demonstrate that there are no coherent spin fluctuations taken into account.

\section{B. Non-crossing approximation (NCA)}

As noted before, in the present context, we may take the hybridization $V$ to be a small quantity (dimensionless parameter $\mathcal{N}_{o} V$ ). This suggests to start with the lowest order (in $V$ ) diagram of $\Phi$, which is second order (see Fig. 2). The self-energies generated from this obey after analytic continuation to real frequencies $(i \omega \rightarrow \omega-i 0)$ and projection the following equations of self-consistent second order perturbation theory

$$
\begin{aligned}
\Sigma_{f \sigma}^{(N C A)}(\omega-i 0) & =\Gamma \sum_{\mu} \int \frac{d \varepsilon}{\pi}[1-f(\varepsilon)] A_{c \mu \sigma}^{0}(\varepsilon) G_{b \bar{\mu}}(\omega-\varepsilon-i 0) \\
\Sigma_{b \bar{\mu}}^{(N C A)}(\omega-i 0) & =\Gamma \sum_{\sigma} \int \frac{d \varepsilon}{\pi} f(\varepsilon) A_{c \mu \sigma}^{0}(\varepsilon) G_{f \sigma}(\omega+\varepsilon-i 0) \\
G_{d \mu \sigma}^{(N C A)}(\omega-i 0) & =\int d \varepsilon \mathrm{e}^{-\beta \varepsilon}\left[G_{f \sigma}(\omega+\varepsilon-i 0) A_{b \bar{\mu}}(\varepsilon)-A_{f \sigma}(\varepsilon) G_{b \bar{\mu}}(\varepsilon-\omega+i 0)\right] \\
& =\int d \varepsilon\left[G_{f \sigma}(\omega+\varepsilon-i 0) A_{b \bar{\mu}}^{-}(\varepsilon)-A_{f \sigma}^{-}(\varepsilon) G_{b \bar{\mu}}(\varepsilon-\omega+i 0)\right]
\end{aligned}
$$

where $A_{c \mu \sigma}^{0}=\frac{1}{\pi} \operatorname{Im} G_{c \mu \sigma}^{0} / \mathcal{N}(0)$ is the (free) conduction electron density of states per spin and channel, normalized to the density of states at the Fermi level $\mathcal{N}(0)$, and $f(\varepsilon)=1 /(\exp (\beta \varepsilon)+1)$ denotes the Fermi distribution function. Together with the expressions (21), 23) for the Green's functions, Eqs. (52)-(54) form a set of self-consistent equations for $\Sigma_{b, f, c}$, comprised of all diagrams without any crossing propagator lines and are, thus, known the "non-crossing approximation", in short NCA40.4.

At zero temperature and for low frequencies, Eqs. (52) and (53) may be converted intp a set of linear differential equations for $G_{f}$ and $G_{b} 42$, which allow to find the infrared exponents as $\alpha_{f}=\frac{M}{M+N} ; \alpha_{b}=\frac{N}{M+N}$, independent of $n_{d}$. For the single channel case these exponents do not agree with the exact exponents derived in section III B. This indicates that the NCA is not capable of recovering the local Fermi liquid behavior for $M=1$. A numerical evaluation of the $d$-electron Green's function, which is given by the local self-energy $\Sigma_{c}$ divided by $V^{2}$ and hence is given by the boson-fermion bubble within NCA (Fig. 2), shows indeed a spurious singularity at the
Fermi energy 43 . The NCA performs somewhat better in the multi-channel case, where the exponents $\alpha_{f}$ and $\alpha_{b}$ yield the correct non-Fermi liquid exponents of physical quantities as known from the Bethe ansatz solution 15 and conformal field theory 19 . However, the specific heat and the residual entropy are not given correctly in NCA. Also, the limiting low temperature scaling laws for the thermodynamic quantities are attained only at temperatures substantially below $T_{K}$, in disagreement with the exact Bethe ansatz solution.

\section{Evaluation of the self-consistency equations at low temperatures}

In order to enter the asymptotic power law regime of the auxiliary spectral functions, the self-consistent equations must be evaluated for temperatures several orders of magnitude below $T_{K}$, the low temperature scale of the model. The equations are solved numerically by iteration. In the following we describe the two main procedures to make the diagrammatic auxiliary particle tech- 
nique suitable for the lowest temperatures.

The grand-canonical expectation value of the auxiliary particle number appearing in Eq. (18) is given in terms of the grand-canonical (unprojected) auxiliary particle spectral functions $\mathcal{A}_{f, b}(\omega, \lambda)$ by,

$$
\begin{aligned}
& \langle Q\rangle_{G}(\lambda)= \\
& \int d \omega\left[f(\omega) \sum_{\sigma} \mathcal{A}_{f \sigma}(\omega, \lambda)+b(\omega) \sum_{\mu} \mathcal{A}_{b \bar{\mu}}(\omega, \lambda)\right]
\end{aligned}
$$

where $f(\omega), b(\omega)$ are the Fermi and Bose distribution functions, respectively. Substituting this into the expression (16) for the canonical partition function we obtain after carrying out the transformation $\omega \rightarrow \omega+\lambda$, and taking the limit $\lambda \rightarrow \infty$

$$
\begin{aligned}
e^{-\beta F_{i m p}(T)} & \equiv \frac{Z_{C}}{Z_{Q=0}}=\lim _{\lambda \rightarrow \infty} e^{\beta \lambda}\langle Q\rangle_{G}(\lambda) \\
& =\int d \omega e^{-\beta \omega}\left[\sum_{\sigma} A_{f \sigma}(\omega)+\sum_{\mu} A_{b \bar{\mu}}(\omega)\right] .
\end{aligned}
$$

By definition $F_{i m p}=-\frac{1}{\beta} \ln \left(Z_{C} / Z_{Q=0}\right)$ is the impurity contribution to the Free energy.

The numerical evaluation of expectation values like $\langle Q\rangle_{G}(\lambda \rightarrow \infty)$ (Eq. (56)) or $G_{d \mu \sigma}(\omega, \lambda \rightarrow \infty)$ (Eq. (54)) is non-trivial, (1) because at $T=0$ the auxiliary spectral functions $A_{f, b}(\omega, T)$ are divergent at the threshold frequency $E_{0}$, where the exact position of $E_{0}$ is a priori not known, and (2) because the Boltzmann factors $e^{-\beta \omega}$ diverge exponentially for $\omega<0$. Therefore, we apply the following transformations:

(1) Before performing the projection $\omega \rightarrow \omega+\lambda$, $\lambda \rightarrow \infty$ we re-define the frequency scale of all auxiliary particle functions $A_{f, b}$ according to $\omega \rightarrow \omega+\lambda_{0}$, where $\lambda_{0}$ is a finite parameter. In each iteration $\lambda_{0}$ is then determined such that

$$
\int d \omega e^{-\beta \omega}\left[\sum_{\sigma} A_{f \sigma}(\omega)+\sum_{\mu} A_{b \bar{\mu}}(\omega)\right]=1
$$

where $A_{f, b}(\omega)=\lim _{\lambda \rightarrow \infty} A_{f, b}\left(\omega+\lambda_{0}, \lambda\right)$ is now an auxiliary spectral function with the new reference energy.
It is seen by comparison with Eq. (56) that $\lambda_{0}(T)=$ $F_{i m p}(T)=F_{Q=1}(T)-F_{Q=0}(T)$, i.e. $\lambda_{0}$ is the chemical potential for the auxiliary particle number $Q$, or equivalently the impurity contribution to the Free energy. The difference of the Free energies becomes equal to the threshold energy $E_{0}=E_{Q=1}^{G S}-E_{Q=0}^{G S}$ at $T=0$. More importantly, however, the above way of determining a "threshold" is less ad hoc than, for example, defining it by a maximum in some function appearing in the NCA equations. It is also seen from Eq. (57) that this procedure defines the frequency scale of the auxiliary particles such that the $T=0$ threshold divergence of the spectral functions is at the fixed frequency $\omega=0$. This substantially increases the precision as well as the speed of numerical evaluations. Eq. (18) for the projected $d-$ electron Green's function becomes

$$
G_{d}(\omega)=\lim _{\lambda \rightarrow \infty} e^{\beta \lambda} G_{d}(\omega, T, \lambda) .
$$

(2) The divergence of the Boltzmann factors implies that the self-consistent solutions for $A_{f, b}(\omega)$ vanish exponentially $\sim e^{\beta \omega}$ for negative frequencies, confirming their threshold behavior. It is convenient, not to formulate the self-consistent equations in terms of $A_{f, b}$ like in earlier evaluations 44 , but to define new functions $\tilde{A}_{f, b}(\omega)$ and $\operatorname{Im} \tilde{\Sigma}_{f, b}(\omega)$ such that

$$
\begin{aligned}
A_{f, b}(\omega) & =f(-\omega) \tilde{A}_{f, b}(\omega) \\
\operatorname{Im} \Sigma_{f, b}(\omega) & =f(-\omega) \operatorname{Im} \tilde{\Sigma}_{f, b}(\omega) .
\end{aligned}
$$

After fixing the chemical potential $\lambda_{0}$ and performing the projection onto the physical subspace, the canonical partition function (Eq. (16) ) behaves as $\lim _{\lambda \rightarrow \infty} e^{\beta\left(\lambda-\lambda_{0}\right)} Z_{C}(T)=1$, and from Eq. (43) we have $A_{f, b}^{-}(\omega)=f(\omega) \tilde{A}_{f, b}(\omega)$. In this way all exponential divergencies are absorbed by one single function for each particle species. As an example, the NCA equations in terms of these functions are free of divergencies of the statistical factors and read

$$
\begin{aligned}
\operatorname{Im} \tilde{\Sigma}_{f \sigma}(\omega-i 0) & =\Gamma \sum_{\mu} \int d \varepsilon \frac{f(-\varepsilon)(1-f(\omega-\varepsilon))}{1-f(\omega)} A_{c \mu \sigma}^{0}(\varepsilon) \tilde{A}_{b \bar{\mu}}(\omega-\varepsilon) \\
\operatorname{Im} \tilde{\Sigma}_{b \bar{\mu}}(\omega-i 0) & =\Gamma \sum_{\sigma} \int d \varepsilon \frac{f(\varepsilon)(1-f(\omega+\varepsilon))}{1-f(\omega)} A_{c \mu \sigma}^{0}(\varepsilon) \tilde{A}_{f \sigma}(\omega+\varepsilon) \\
\langle Q\rangle\left(\lambda_{0}, \lambda \rightarrow \infty\right) & =\int d \omega f(\omega)\left[\sum_{\sigma} \tilde{A}_{f \sigma}(\omega)+\sum_{\mu} \tilde{A}_{b \bar{\mu}}(\omega)\right]=1 \\
\operatorname{Im} G_{d \sigma}(\omega-i 0) & =\int d \varepsilon[f(\varepsilon+\omega) f(-\varepsilon)+f(-\varepsilon-\omega) f(\varepsilon)] \tilde{A}_{f \sigma}(\varepsilon+\omega) \tilde{A}_{b}(\varepsilon) .
\end{aligned}
$$



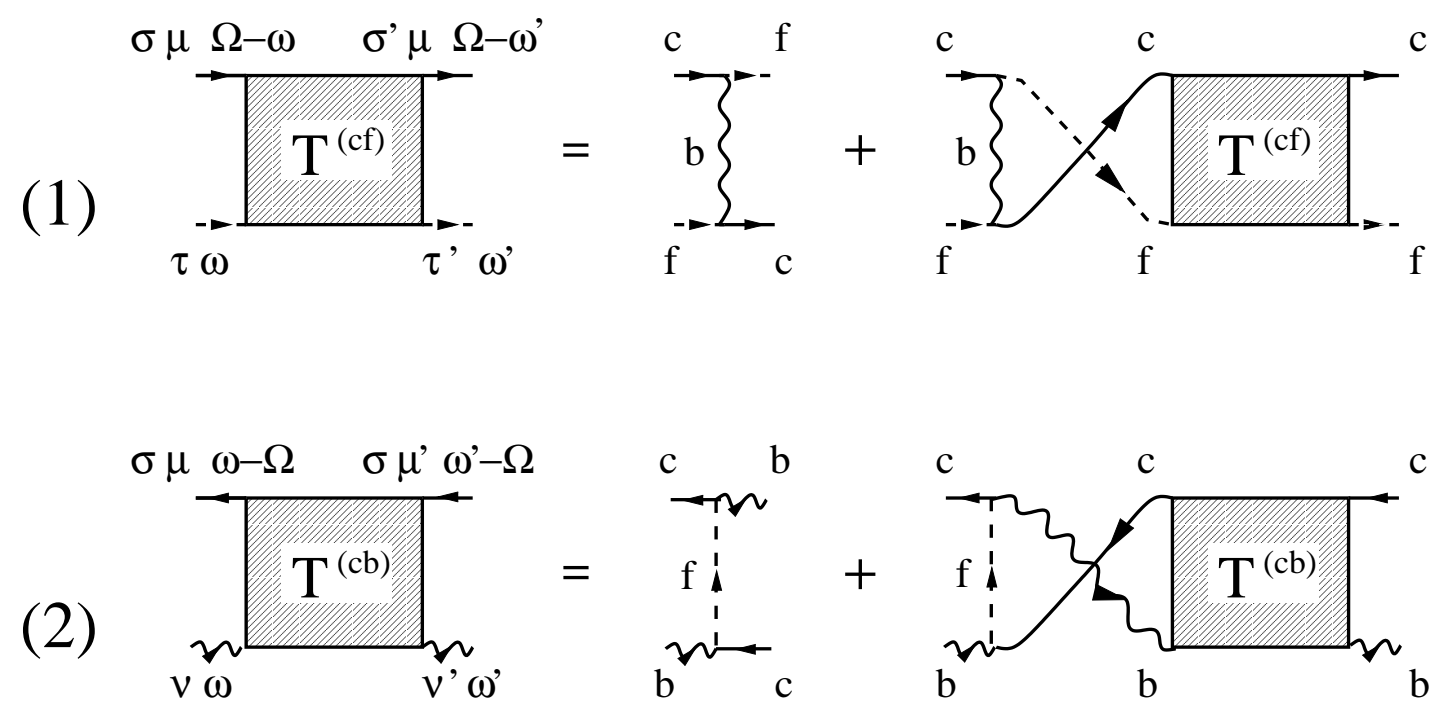

FIG. 3. Diagrammatic representation of the Bethe-Salpeter equation for (1) the conduction electron-pseudofermion T-matrix $T^{(c f)}$, Eq. $(65)$, and (2) the conduction electron-slave boson T-matrix $T^{(c b)}$, Eq. (66). $T^{(c b)}$ is obtained from $T^{(c f)}$ by interchanging $f \leftrightarrow b$ and $c \leftrightarrow c^{\dagger}$.

The real parts of the self-energies $\Sigma_{f}, \Sigma_{b}$ are determined from $\operatorname{Im} \Sigma_{f}, \operatorname{Im} \Sigma_{b}$ through a Kramers-Kroenig relation, and the auxiliary functions $\tilde{A}_{f \sigma}(\omega)=\frac{1}{\pi} \operatorname{Im} \tilde{\Sigma}_{f \sigma}(\omega-$ $i 0) /\left[\left(\omega+\lambda_{0}-i 0-E_{d}-\operatorname{Re} \Sigma_{f \sigma}(\omega-i 0)\right)^{2}+\operatorname{Im} \Sigma_{f \sigma}(\omega-i 0)^{2}\right]$, $\tilde{A}_{b \bar{\mu}}(\omega)=\frac{1}{\pi} \operatorname{Im} \tilde{\Sigma}_{b \bar{\mu}}(\omega-i 0) /\left[\left(\omega+\lambda_{0}-i 0-\operatorname{Re} \Sigma_{b \bar{\mu}}(\omega-\right.\right.$ $\left.i 0))^{2}+\operatorname{Im} \Sigma_{b \bar{\mu}}(\omega-i 0)^{2}\right]$, thus closing the above set of equations.

The method described above allows to solve the NCA equations effectively for temperatures down to typically $T=10^{-4} T_{K}$. It may be shown that the procedures described above can also be applied to self-consistently compute vertex corrections beyond the NCA (see section $\mathrm{VI}$ ), thus avoiding any divergent statistical factors in the selfconsistency equations.

\section{CONSERVING T-MATRIX APPROXIMATION (CTMA) AT $U \rightarrow \infty$}

\section{A. Dominant contributions at low energy}

In order to eliminate the shortcomings of the NCA mentioned above, the guiding principle should be to find contributions to the vertex functions which renormalize the auxiliary particle threshold exponents to their correct values, since this is a necessary condition for the description of FL and non-FL behavior, as discussed in section III B. Furthermore, it is instructive to realize that in
NCA any coherent spin flip and charge transfer processes are neglected, as can be seen explicitly from Eqs. (52), (53) or from Fig. 2. These processes are known to be responsible for the quantum coherent collective behavior of the Anderson impurity complex below $T_{K}$. The existence of collective excitations in general is reflected in a singular behavior of the corresponding two-particle vertex functions. In view of the tendency of Kondo systems to form a collective spin singlet state, we focus our attention on the two-particle vertex functions, in particular, in the spin singlet channel of the pseudofermion-conduction electron vertex function and in the slave boson-conduction electron vertex function. It may be shown by power counting arguments (compare appendix A) that there are no corrections to the NCA exponents in any finite order of perturbation theory 45 . Thus, we are led to search for singularities in the aforementioned vertex functions arising from an infinite resummation of terms.

From the preceding discussion it is natural to perform a partial resummation of those terms which, at each order in the hybridization $V$, contain the maximum number of spin flip or charge fluctuation processes, respectively. This amounts to calculating the conduction electronpseudofermion vertex function in the "ladder" approximation defined in Fig. 3, where the irreducible vertex is given by $V^{2} G_{b}$. In analogy to similar resummations for an interacting one-component Fermi system, we call the total $\mathrm{c}-\mathrm{f}$ vertex function $\mathrm{T}$-matrix $T^{(c f)}$. The BetheSalpeter equation for $T^{(c f)}$ reads (Fig. 通(1)),

$$
\begin{aligned}
T_{\sigma \tau, \sigma^{\prime} \tau^{\prime}}^{(c f) \mu}\left(i \omega_{n}, i \omega_{n}^{\prime}, i \Omega_{n}\right)= & +V^{2} G_{b \bar{\mu}}\left(i \omega_{n}+i \omega_{n}^{\prime}-i \Omega_{n}\right) \delta_{\sigma \tau^{\prime}} \delta_{\tau \sigma^{\prime}} \\
& -V^{2} T \sum_{\omega_{n}^{\prime \prime}} G_{b \bar{\mu}}\left(i \omega_{n}+i \omega_{n}^{\prime \prime}-i \Omega_{n}\right) G_{f \sigma}\left(i \omega_{n}^{\prime \prime}\right) G_{c \mu \tau}^{0}\left(i \Omega_{n}-i \omega_{n}^{\prime \prime}\right) T_{\tau \sigma, \sigma^{\prime} \tau^{\prime}}^{(c f) \mu}\left(i \omega_{n}^{\prime \prime}, i \omega_{n}^{\prime}, i \Omega_{n}\right) .
\end{aligned}
$$


A similar integral equation holds for the charge fluctuation T-matrix $T^{(c b)}$ (Fig. $3(2)$ ),

$$
\begin{aligned}
T_{\mu \nu, \mu^{\prime} \nu^{\prime}}^{(c b) \sigma}\left(i \omega_{n}, i \omega_{n}^{\prime}, i \Omega_{n}\right)= & +V^{2} G_{f \sigma}\left(+i \omega_{n}+i \omega_{n}^{\prime}-i \Omega_{n}\right) \delta_{\mu \nu^{\prime}} \delta_{\nu \mu^{\prime}} \\
& -V^{2} T \sum_{\omega_{n}^{\prime \prime}} G_{f \sigma}\left(i \omega_{n}+i \omega_{n}^{\prime \prime}-i \Omega_{n}\right) G_{b \bar{\mu}}\left(i \omega_{n}^{\prime \prime}\right) G_{c \nu \sigma}^{0}\left(-i \omega_{n}^{\prime \prime}-i \Omega_{n}\right) T_{\nu \mu, \mu^{\prime} \nu^{\prime}}^{(c b) \sigma}\left(i \omega_{n}^{\prime \prime}, i \omega_{n}^{\prime}, i \Omega_{n}\right) .
\end{aligned}
$$

In the above Bethe-Salpeter equations $\sigma, \tau, \sigma^{\prime}, \tau^{\prime}$ represent spin and $\mu, \nu, \mu^{\prime}, \nu^{\prime}$ channel indices. We note that these are the only two-particle vertex functions after projection. The principal approximation adopted here is the form of the irreducible kernel, which we approximate by the lowest order diagram.

Inserting NCA Green's functions for the intermediate state propagators of Eq. (65) and solving it numerically, we find at low temperatures and in the Kondo regime $\left(n_{d} \gtrsim 0.7\right)$ a pole of $T^{(c f)}$ in the singlet channel (see appendix A) as a function of the center-of-mass (COM) frequency $\Omega$, at a frequency which scales with the Kondo temperature, $\Omega=\Omega_{c f} \simeq-T_{K}$. This is shown in Fig. 4 . The threshold behavior of the imaginary part of $T^{(c f)}$ as a function of $\Omega$ with vanishing spectral weight at negative frequencies and temperature $T=0$ is clearly seen. In addition, a very sharp structure appears, whose broadening is found to vanish as the temperature tends to zero, indicative of a pole in $T^{(c f)}$ at the real frequency $\Omega_{c f}$, i.e. the tendency to form a collective singlet state between the conduction electrons and the localized spin.

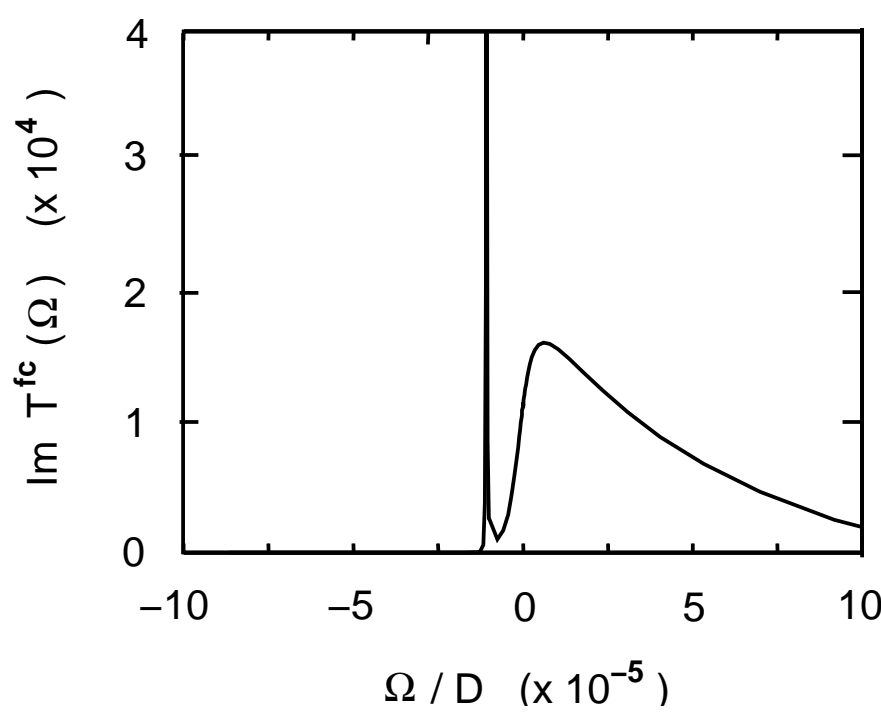

FIG. 4. Imaginary part of the conduction electron-pseudofermion T-matrix $T^{(c f)}$ as a function of the COM frequency $\Omega$ for the single-channel case $M=1, N=2$, evaluated by inserting NCA solutions for the intermediate state propagators $\left(E_{d}=-0.67 D, \Gamma=0.15 D, T=4 \cdot 10^{-3} T_{K}\right)$. The contribution from the pole positioned at a negative frequency $\Omega=\Omega_{c f} \simeq-T_{K}$ (compare text) is clearly seen.

Similarly, the corresponding $T$-matrix $T^{(c b)}$ in the con- duction electron-slave boson channel, evaluated within the analogous approximation, develops a pole at negative values of $\Omega$ in the empty orbital regime $\left(n_{d} \lesssim 0.3\right)$. In the mixed valence regime $\left(n_{d} \simeq 0.5\right)$ the poles in both $T^{(c f)}$ and $T^{(c b)}$ coexist. The appearance of poles in the two-particle vertex functions $T^{(c f)}$ and $T^{(c b)}$, which signals the formation of collective states, may be expected to influence the behavior of the system in a major way.

\section{B. Self-consistent formulation: CTMA}

On the level of approximation considered so far, the description is not yet consistent: In the limit of zero temperature the spectral weight of $T^{(c f)}$ and $T^{(c b)}$ at negative frequencies $\Omega$ should be strictly zero (threshold property). Nonvanishing spectral weight at $\Omega<0$ like a pole contribution for negative $\Omega$ in $T^{(c f)}$ or $T^{(c b)}$ would lead to a diverging contribution to the self-energy, which is unphysical. However, recall that a minimum requirement on the approximation used is the preservation of gauge symmetry. This requirement is not met when the integral kernel of the $T$-matrix equation is approximated by the NCA result. Rather, the approximation should be generated from a Luttinger-Ward functional. The corresponding generating functional is shown in Fig. 5. It is defined as the infinite series of all vacuum skeleton diagrams which consist of a single ring of auxiliary particle propagators, where each conduction electron line spans at most two hybridization vertices. As shown in appendix A by means of a cancellation theorem, the CTMA includes, at any given loop order, all infrared singular contributions to leading and subleading order in the frequency $\omega$. The first diagram of the infinite series of CTMA terms corresponds to NCA (Fig. 2). The diagram containing two boson lines is excluded, since it is not a skeleton. Although the spirit of the present theory is different from a large $N$ expansion, it should be noted that the sum of the $\Phi$ diagrams containing up to four boson lipes includes all terms of a $1 / N$ expansion up to $O\left(1 / N^{2}\right) 4$. By functional differentiation with respect to the conduction electron Green's function and the pseudofermion or the slave boson propagator, respectively, the shown $\Phi$ functional generates the ladder approximations $T^{(c f)}, T^{(c b)}$ 


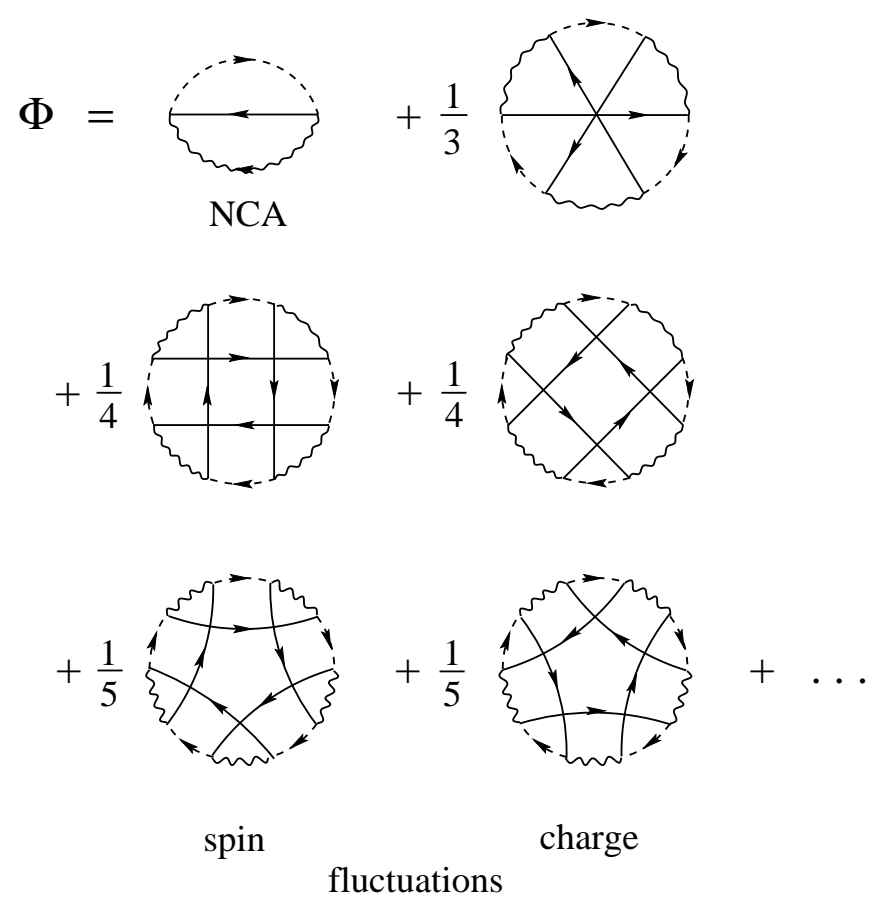

FIG. 5. Diagrammatic representation of the Luttinger-Ward functional generating the conserving $\mathrm{T}$-matrix approximation (CTMA). The terms with the conduction electron lines running clockwise (labeled "spin fluctuations") generate the T-matrix $T^{(c f)}$, while the terms with the conduction electron lines running counter-clockwise (labeled "charge fluctuations") generate the T-matrix $T^{(c b)}$. for the total conduction electron-pseudofermion vertex function and for the total conduction electron-slave boson vertex function (Fig. 3). The auxiliary particle self-energies are obtained in the conserving scheme as the functional derivatives of $\Phi$ with respect to $G_{f}$ or $G_{b}$, respectively (Eq. (51)). This defines a set of self-consistency equations, which we term conserving $\mathrm{T}-$ matrix approximation (CTMA), where the self-energies are given as nonlinear and nonlocal (in time) functionals of the Green's functions, while the Green's functions are in turn expressed in terms of the self-energies. The solution of these equations requires that the $\mathrm{T}$-matrices have vanishing spectral weight at negative COM frequencies $\Omega$. Indeed, the numerical evaluation shows that the poles of $T^{(c f)}$ and $T^{(c b)}$ are shifted to $\Omega=0$ by selfconsistency, where they merge with the continuous spectral weight present for $\Omega>0$, thus renormalizing the threshold exponents of the auxiliary spectral functions.

In the following we give explicitly the self-consistent equations which determine the auxiliary particle selfenergies within CTMA. For that purpose, it is useful to define conduction electron-fermion and conduction electron-boson vertex functions $T^{(c f)( \pm)}, T^{(c b)( \pm)}$ without $(+)$ or with $(-)$ an alternating sign between terms with even and odd number of rungs (compare Fig. 3). In the Matsubara representation, these vertex functions, to be labelled "even" $(+)$ and "odd" $(-)$ below, are given by the following Bethe-Salpeter equations:

$$
\begin{aligned}
T_{\sigma, \tau}^{(c f)( \pm) \mu}\left(i \omega_{n}, i \omega_{n}^{\prime}, i \Omega_{n}\right) & =U_{\sigma, \tau}^{(c f) \mu}\left(i \omega_{n}, i \omega_{n}^{\prime}, i \Omega_{n}\right) \\
& \pm V^{2} \frac{1}{\beta} \sum_{\omega_{n}^{\prime \prime}} G_{b \bar{\mu}}\left(i \omega_{n}+i \omega_{n}^{\prime \prime}-i \Omega_{n}\right) G_{f \sigma}\left(i \omega_{n}^{\prime \prime}\right) G_{c \mu \tau}^{0}\left(i \Omega_{n}-i \omega_{n}^{\prime \prime}\right) T_{\tau, \sigma}^{(c f)( \pm) \mu}\left(i \omega_{n}^{\prime \prime}, i \omega_{n}^{\prime}, i \Omega_{n}\right) \\
U_{\sigma, \tau}^{(c f) \mu}\left(i \omega_{n}, i \omega_{n}^{\prime}, i \Omega_{n}\right) & =-V^{4} \frac{1}{\beta} \sum_{\omega_{n}^{\prime \prime}} G_{b \bar{\mu}}\left(i \omega_{n}+i \omega_{n}^{\prime \prime}-i \Omega_{n}\right) G_{f \sigma}\left(i \omega_{n}^{\prime \prime}\right) G_{c \mu \tau}^{0}\left(i \Omega_{n}-i \omega_{n}^{\prime \prime}\right) G_{b \bar{\mu}}\left(i \omega_{n}^{\prime}+i \omega_{n}^{\prime \prime}-i \Omega_{n}\right)
\end{aligned}
$$

and

$$
\begin{aligned}
T_{\mu, \nu}^{(c b)( \pm) \sigma}\left(i \omega_{n}, i \omega_{n}^{\prime}, i \Omega_{n}\right) & =U_{\mu, \nu}^{(c b) \sigma}\left(i \omega_{n}, i \omega_{n}^{\prime}, i \Omega_{n}\right) \\
& \pm V^{2} \frac{1}{\beta} \sum_{\omega_{n}^{\prime \prime}} G_{f \sigma}\left(i \omega_{n}+i \omega_{n}^{\prime \prime}-i \Omega_{n}\right) G_{b \bar{\mu}}\left(i \omega_{n}^{\prime \prime}\right) G_{c \nu \sigma}^{0}\left(i \omega_{n}^{\prime \prime}-i \Omega_{n}\right) T_{\nu, \mu}^{(c b)( \pm) \sigma}\left(i \omega_{n}^{\prime \prime}, i \omega_{n}^{\prime}, i \Omega_{n}\right) \\
U_{\mu, \nu}^{(c b) \sigma}\left(i \omega_{n}, i \omega_{n}^{\prime}, i \Omega_{n}\right) & =-V^{4} \frac{1}{\beta} \sum_{\omega_{n}^{\prime \prime}} G_{f \sigma}\left(i \omega_{n}+i \omega_{n}^{\prime \prime}-i \Omega_{n}\right) G_{b \bar{\mu}}\left(i \omega_{n}^{\prime \prime}\right) G_{c \nu \sigma}^{0}\left(i \omega_{n}^{\prime \prime}-i \Omega_{n}\right) G_{f \sigma}\left(i \omega_{n}^{\prime}+i \omega_{n}^{\prime \prime}-i \Omega_{n}\right) .
\end{aligned}
$$

Note that, in addition to the alternating sign, these vertex functions differ from the $\mathrm{T}$-matrices defined in Eqs. (65), (66) in that they contain only terms with two or more rungs, since the inhomogeneous parts $U^{(c f)}$ and $U^{(c b)}$ represent terms with two bosonic or fermionic rungs, respectively. The terms with a single rung corre- spond to the NCA diagrams and are evaluated separately (see below).

The spin degrees of freedom of $T^{(c f)( \pm)}$ are uniquely determined by the spin indices $\sigma, \tau$ of the ingoing conduction electron and pseudofermion lines (Fig. 3). It is 


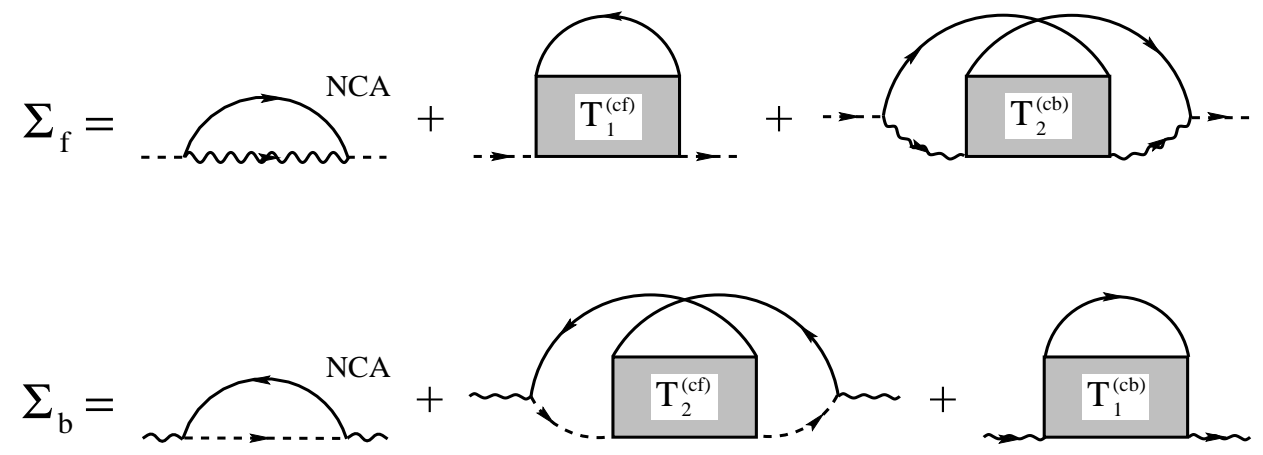

FIG. 6. Diagrammatic representation of the CTMA expressions for pseudoparticle self-energies $\Sigma_{f}$ and $\Sigma_{b}$. The first term drawn on the righthand side of $\Sigma_{f}$ and $\Sigma_{b}$, respectively, is the NCA diagram. The diagrammatic parts $T_{1,2}^{(c f)}, T_{1,2}^{(c b)}$ are explained in the text, Eqs. (80)-(83).

instructive to note that in the $\operatorname{spin} S=1 / 2$ case $(N=2)$ the singlet and triplet vertex functions (which correspond to the two-particle Green's functions in the singlet channel, $\phi^{s} \sim \sum_{\sigma}\left\langle T\left\{\left(c_{\sigma} f_{-\sigma}-c_{-\sigma} f_{\sigma}\right)\left(c_{\sigma}^{\dagger} f_{-\sigma}^{\dagger}-c_{-\sigma}^{\dagger} f_{\sigma}^{\dagger}\right)\right\}\right\rangle$, and in the triplet channel with magnetic quantum number $m=0, \pm 1, \phi_{m=0}^{t} \sim \sum_{\sigma}\left\langle T\left\{\left(c_{\sigma} f_{-\sigma}+c_{-\sigma} f_{\sigma}\right)\left(c_{\sigma}^{\dagger} f_{-\sigma}^{\dagger}+\right.\right.\right.$ $\left.\left.\left.c_{-\sigma}^{\dagger} f_{\sigma}^{\dagger}\right)\right\}\right\rangle, \phi_{m= \pm 1}^{t} \sim\left\langle T\left\{c_{ \pm \frac{1}{2}} f_{ \pm \frac{1}{2}} c_{ \pm \frac{1}{2}}^{\dagger} f_{ \pm \frac{1}{2}}^{\dagger}\right\}\right\rangle$, respectively) may be identified in the following way,

$$
\begin{aligned}
& T^{(c f) s}=\sum_{\sigma} T_{\sigma,-\sigma}^{(c f)(-)} \\
& T_{m=0}^{(c f) t}=\sum_{\sigma} T_{\sigma,-\sigma}^{(c f)(+)} \\
& T_{m= \pm 1}^{(c f) t}=\quad T_{ \pm \frac{1}{2}, \pm \frac{1}{2}}^{(c f)(+)} .
\end{aligned}
$$

Analogous relations hold for the conduction electron- boson vertex function in terms of the channel degrees of freedom $\mu, \nu$. The total CTMA pseudoparticle selfenergies, as derived by functional differentiation from the generating functional $\Phi$, Fig. 5, are shown in Fig. 6 and consist of three terms each,

$$
\begin{aligned}
& \Sigma_{f \sigma}\left(i \omega_{n}\right)=\Sigma_{f \sigma}^{(N C A)}\left(i \omega_{n}\right)+\Sigma_{f \sigma}^{(c f)}\left(i \omega_{n}\right)+\Sigma_{f \sigma}^{(c b)}\left(i \omega_{n}\right) \\
& \Sigma_{b \bar{\mu}}\left(i \omega_{n}\right)=\Sigma_{b \bar{\mu}}^{(N C A)}\left(i \omega_{n}\right)+\Sigma_{b \bar{\mu}}^{(c f)}\left(i \omega_{n}\right)+\Sigma_{b \bar{\mu}}^{(c b)}\left(i \omega_{n}\right) .
\end{aligned}
$$

The first term of $\Sigma_{f}$ and $\Sigma_{b}$ represents the NCA selfenergies, Eqs. (52), (53). The second and third terms arise from the spin and the charge fluctuations, respectively, and are given for pseudofermions by

$$
\begin{aligned}
& \Sigma_{f \sigma}^{(c f)}\left(i \omega_{n}\right)=M \frac{1}{\beta} \sum_{\Omega_{n}} G_{c}^{0}\left(i \Omega_{n}-i \omega_{n}\right) T_{1}^{(c f)}\left(i \omega_{n}, i \omega_{n}, i \Omega_{n}\right) \\
& \Sigma_{f \sigma}^{(c b)}\left(i \omega_{n}\right)=-M V^{2} \frac{1}{\beta^{2}} \sum_{\omega_{n}^{\prime} \omega_{n}^{\prime \prime}} G_{c}^{0}\left(i \omega_{n}-i \omega_{n}^{\prime}\right) G_{b}\left(i \omega_{n}^{\prime}\right) T_{2}^{(c b)}\left(i \omega_{n}^{\prime}, i \omega_{n}^{\prime \prime}, i \omega_{n}^{\prime}+i \omega_{n}^{\prime \prime}-i \omega_{n}\right) G_{c}^{0}\left(i \omega_{n}-i \omega_{n}^{\prime \prime}\right) G_{b}\left(i \omega_{n}^{\prime \prime}\right)
\end{aligned}
$$

and for slave bosons by

$$
\begin{aligned}
& \Sigma_{b \bar{\mu}}^{(c f)}\left(i \omega_{n}\right)=-N V^{2} \frac{1}{\beta^{2}} \sum_{\omega_{n}^{\prime} \omega_{n}^{\prime \prime}} G_{c}^{0}\left(i \omega_{n}^{\prime}-i \omega_{n}\right) G_{f}\left(i \omega_{n}^{\prime}\right) T_{2}^{(c f)}\left(i \omega_{n}^{\prime}, i \omega_{n}^{\prime \prime}, i \omega_{n}^{\prime}+i \omega_{n}^{\prime \prime}-i \omega_{n}\right) G_{c}^{0}\left(i \omega_{n}^{\prime \prime}-i \omega_{n}\right) G_{f}\left(i \omega_{n}^{\prime \prime}\right) \\
& \Sigma_{b \bar{\mu}}^{(c b)}\left(i \omega_{n}\right)=N \frac{1}{\beta} \sum_{\Omega_{n}} G_{c}^{0}\left(i \omega_{n}-i \Omega_{n}\right) T_{1}^{(c b)}\left(i \omega_{n}, i \omega_{n}, i \Omega_{n}\right)
\end{aligned}
$$

where the vertex functions appearing in these expressions are defined as

$$
\begin{aligned}
& T_{1}^{(c f)}=\frac{N+1}{2} T^{(c f)(+)}+\frac{N-1}{2} T^{(c f)(-)}-N U^{(c f)} \\
& T_{2}^{(c f)}=\frac{N+1}{2} T^{(c f)(+)}-\frac{N-1}{2} T^{(c f)(-)}-U^{(c f)}
\end{aligned}
$$




$$
\begin{aligned}
& T_{1}^{(c b)}=\frac{M-1}{2} T^{(c b)(+)}+\frac{M+1}{2} T^{(c b)(-)}-M U^{(c b)} \\
& T_{2}^{(c b)}=\frac{M-1}{2} T^{(c b)(+)}-\frac{M+1}{2} T^{(c b)(-)}-U^{(c b)} .
\end{aligned}
$$

These combinations of the even and odd vertex functions ensure the proper spin and channel summations in the self-energies. For the sake of clarity, the spin and channel indices as well as the frequency variables are not shown explicitly. In Eqs. 800, (82) the terms with two rungs, $N U^{(c f)}, M U^{(c b)}$, have been subtracted, since they would generate non-skeleton self-energy diagrams. Likewise, in Eqs. (81), (83) the two-rung terms have been subtracted in order to avoid a double counting of terms in the selfenergies.

We now turn to the analytic continuation to real frequencies of the expressions derived above. Transforming the Matsubara summations into contour integrals shows that integrations along branch cuts of auxiliary particle Green's functions carry an additional factor $\exp (-\beta \lambda)$ as compared to integrations along branch cuts of physical Green's functions, which vanishes upon projection onto the the physical Fock space, $\lambda \rightarrow \infty$. Thus, as a general rule, only integrations along branch cuts of the $c$-electron propagators contribute to the auxiliary particle self-energies. Therefore, by performing the analytic continuation, $i \omega_{n} \rightarrow \omega-i 0 \equiv \omega$ in all frequency variables, we obtain the advanced pseudofermion self-energy,

$$
\begin{aligned}
& \Sigma_{f \sigma}^{(c f)}(\omega)=M \int \frac{d \varepsilon}{\pi} f(\varepsilon-\omega) A_{c}^{0}(\varepsilon-\omega) \pi \mathcal{N}(0) T_{1}^{(c f)}(\omega, \omega, \varepsilon) \\
& \Sigma_{f \sigma}^{(c b)}(\omega)=-M \Gamma \int \frac{d \varepsilon}{\pi} \int \frac{d \varepsilon^{\prime}}{\pi} f(\varepsilon-\omega) f\left(\varepsilon^{\prime}-\omega\right) A_{c}^{0}(\omega-\varepsilon) G_{b}(\varepsilon) \pi \mathcal{N}(0) T_{2}^{(c b)}\left(\varepsilon, \varepsilon^{\prime}, \varepsilon+\varepsilon^{\prime}-\omega\right) A_{c}^{0}\left(\omega-\varepsilon^{\prime}\right) G_{b}\left(\varepsilon^{\prime}\right)
\end{aligned}
$$

and the advanced slave boson self-energy,

$$
\begin{aligned}
& \Sigma_{b \bar{\mu}}^{(c f)}(\omega)=-N \Gamma \int \frac{d \varepsilon}{\pi} \int \frac{d \varepsilon^{\prime}}{\pi} f(\varepsilon-\omega) f\left(\varepsilon^{\prime}-\omega\right) A_{c}^{0}(\varepsilon-\omega) G_{f}(\varepsilon) \pi \mathcal{N}(0) T_{2}^{(c f)}\left(\varepsilon, \varepsilon^{\prime}, \varepsilon+\varepsilon^{\prime}-\omega\right) A_{c}^{0}\left(\varepsilon^{\prime}-\omega\right) G_{f}\left(\varepsilon^{\prime}\right) \\
& \Sigma_{b \bar{\mu}}^{(c b)}(\omega)=-N \int \frac{d \varepsilon}{\pi} f(\varepsilon-\omega) A_{c}^{0}(\omega-\varepsilon) \pi \mathcal{N}(0) T_{1}^{(c b)}(\omega, \omega, \varepsilon)
\end{aligned}
$$

where the vertex functions are given by Eqs. (80), 81) and (82), (83) with

$$
\begin{aligned}
T_{\sigma, \tau}^{(c f)( \pm) \mu}\left(\omega, \omega^{\prime}, \Omega\right) & =U^{(c f)( \pm) \mu}\left(\omega, \omega^{\prime}, \Omega\right) \\
& \pm(-\Gamma) \int \frac{d \varepsilon}{\pi} f(\varepsilon-\Omega) G_{b \bar{\mu}}(\omega+\varepsilon-\Omega) G_{f \sigma}(\varepsilon) A_{c \mu \tau}^{0}(\Omega-\varepsilon) T_{\tau, \sigma}^{(c f)( \pm) \mu}\left(\varepsilon, \omega^{\prime}, \Omega\right) \\
\pi \mathcal{N}(0) U_{\sigma, \tau}^{(c f)( \pm) \mu}\left(\omega, \omega^{\prime}, \Omega\right) & =+\Gamma^{2} \int \frac{d \varepsilon}{\pi} f(\varepsilon-\Omega) G_{b \bar{\mu}}(\omega+\varepsilon-\Omega) G_{f \sigma}(\varepsilon) A_{c \mu \tau}^{0}(\Omega-\varepsilon) G_{b \bar{\mu}}\left(\omega^{\prime}+\varepsilon-\Omega\right)
\end{aligned}
$$

and

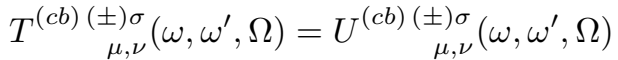

$$
\begin{aligned}
& \pm(+\Gamma) \int \frac{d \varepsilon}{\pi} f(\varepsilon-\Omega) G_{f \sigma}(\omega+\varepsilon-\Omega) G_{b \bar{\mu}}(\varepsilon) A_{c \nu \sigma}^{0}(\varepsilon-\Omega) T_{\nu, \mu}^{(c b)( \pm) \sigma}\left(\omega^{\prime}, \Omega\right) \\
& \pi \mathcal{N}(0) U_{\mu, \nu}^{(c b)( \pm) \sigma}\left(\omega, \omega^{\prime}, \Omega\right)=-\Gamma^{2} \int \frac{d \varepsilon}{\pi} f(\varepsilon-\Omega) G_{f \sigma}(\omega+\varepsilon-\Omega) G_{b \bar{\mu}}(\varepsilon) A_{c \nu \sigma}^{0}(\varepsilon-\Omega) G_{f \sigma}\left(\omega^{\prime}+\varepsilon-\Omega\right)
\end{aligned}
$$

In the above expressions, like in the NCA equations (52)(54), we have used the dimensionless conduction electron spectral density, $A_{c}^{0}(\omega)=\frac{1}{\pi} \operatorname{Im} G_{c \mu \sigma}^{0}(\omega-i 0) / \mathcal{N}(0)$, and we have suppressed obvious spin and channel indices. All frequency variables are to be understood as the limit $\omega \equiv \omega-i 0$.

The equations (84)-(87), supplemented by the vertex functions Eqs. (80)-(83), (88)-(91) form, together with the NCA contributions Eqs. (52), (53) and the definitions of the auxiliary particle Green's functions, Eqs. 21, (23), the closed set of self-consistent CTMA equations 2 . It is seen that in these equations only those branches of the T-matrix vertex functions appear which are advanced with respect to all three frequency variables, although in general the $\mathrm{T}$-matrix consists of $2^{3}$ independent analytical branches. This simplification is a conse- 


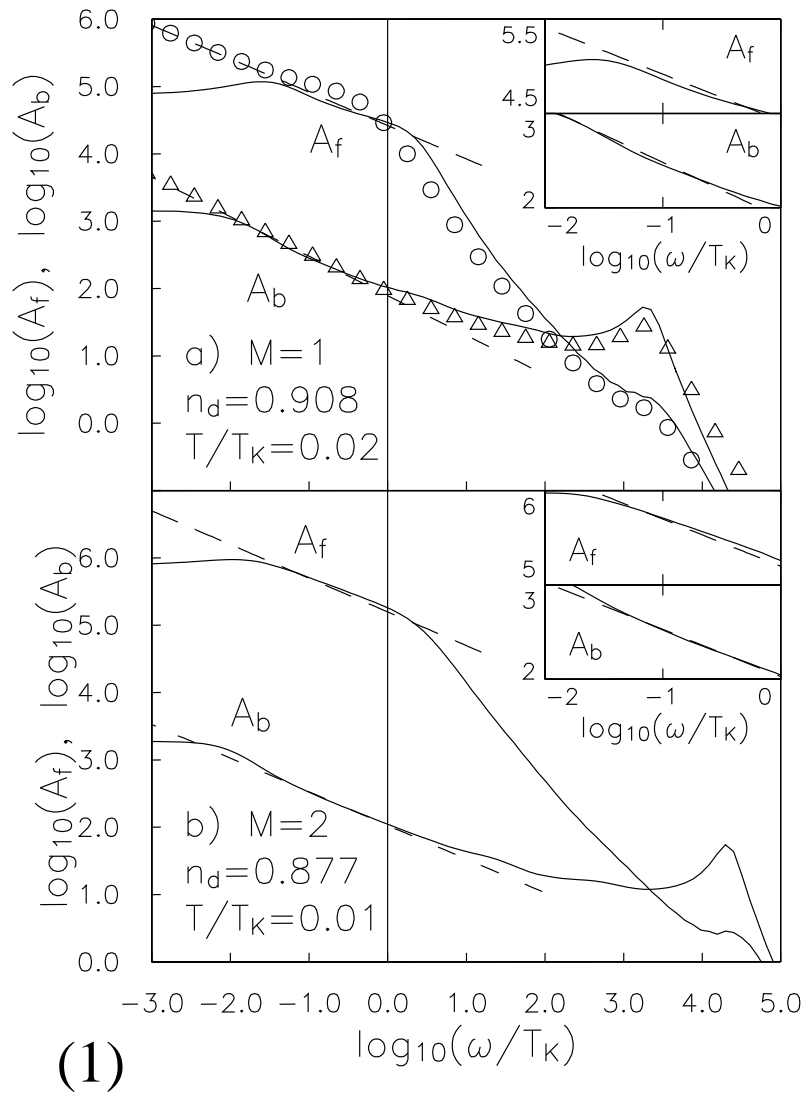

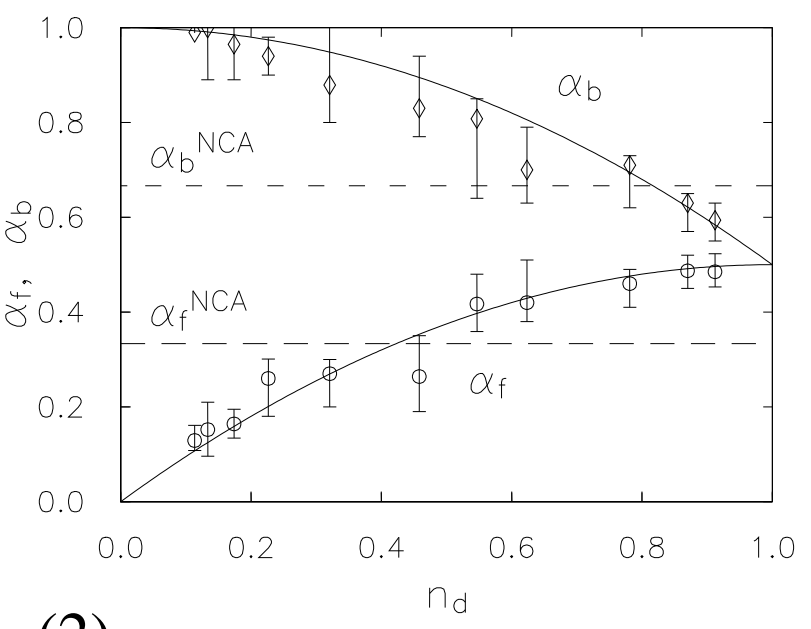

(2)

FIG. 7. (1) Pseudofermion and slave boson spectral functions $A_{f}$ and $A_{b}$ in the Kondo regime $\left(N=2 ; E_{d}=-0.05\right.$, $\Gamma=0.01$ in units of the half-bandwidth $D)$, for a) the single-channel $(M=1)$ and b) the multi-channel $(M=2)$ case. In a) the symbols represent the results of NRG for the same parameter set, $T=0$. The slopes of the dashed lines indicate the exact threshold exponents as derived in section III B for $M=1$ and as given by conformal field theory for $M=2$. The insets show magnified power law regions. (2) CTMA results (symbols with error bars) for the threshold exponents $\alpha_{f}$ and $\alpha_{b}$ of $A_{f}$ and $A_{b},(N=2, M=1)$. Solid lines: exact values (section III B), dashed lines: NCA results (section V B).

quence of the exact projection onto the physical sector of Fock space. Inspection of the analytically continued CTMA equations also shows that the slave boson selfenergy is obtained from the pseudofermion self-energy, including the proper signs, by simply replacing $G_{f} \leftrightarrow G_{b}$ and inverting the frequency argument of $A_{c}^{0}$ in all expressions.

Eqs. (84)-(91) may be rewritten in terms of the spectral functions without threshold, $\tilde{A}_{f, b}$, in a straightforward way as explained in section $\mathrm{V} \mathrm{C}$, thus avoiding divergent statistical factors in the $d$-electron Green's function.

The self-consistent solutions are obtained by first solving the linear Bethe-Salpeter equations for the $\mathrm{T}$ matrices by matrix inversion, computing the auxiliary particle self-energies from $T^{(c f)}$ and $T^{(c b)}$, and then constructing the fermion and boson Green's functions from the respective self-energies. This process is iterated until convergence is reached. We have obtained reliable solutions down to temperatures of the order of at least $10^{-2} T_{K}$ both for the single-channel and for the two-channel Anderson model. Note that $T_{K} \rightarrow 0$ in the Kondo limit; in the mixed valence and empty impurity regimes, significantly lower temperatures may be reached, compared to the low temperature scale of the model.

\section{Results for the auxiliary particle spectral functions}

As shown in Fig. 月(1) a), the amxiliary particle spectral functions obtained from CTMA 4 are in good agreement with the results of a numerical renormalization group (NRG) calculation 28 (zero temperature results), given the uncertainties in the NRG at higher frequencies. Typical behavior in the Kondo regime is obtained: a broad peak of width $\sim \Gamma$ in $A_{b}$ at $\omega \simeq\left|E_{d}\right|$, representing the hybridizing $d$-level and a structure in $A_{f}$ at $\omega \simeq T_{K}$. Both functions display power law behavior at frequen- 
cies below $T_{K}$, which at finite $T$ is cut off at the scale $\omega \simeq T$. The exponents extracted from the frequency range $T<\omega<T_{K}$ of our finite $T$ results compare well with the exact result also shown (see insets of Fig. If (1) a)). A similar analysis has been performed for a number of parameter sets spanning the complete range of $d$-level occupation numbers $n_{d}$. The extracted power law exponents are shown in Fig. 7 (2), together with error bars estimated from the finite frequency ranges over which the fit was made. The comparatively large error bars in the mixed valence regime arise because here spin flip and charge fluctuation processes, described by the poles in $T^{(c f)}$ and $T^{(c b)}$, respectively, are of equal importance, impeding the convergence of the numerical procedure. In this light, the agreement with the exact results (solid curves) is very good, the exact value lying within the error bars or very close in each case.
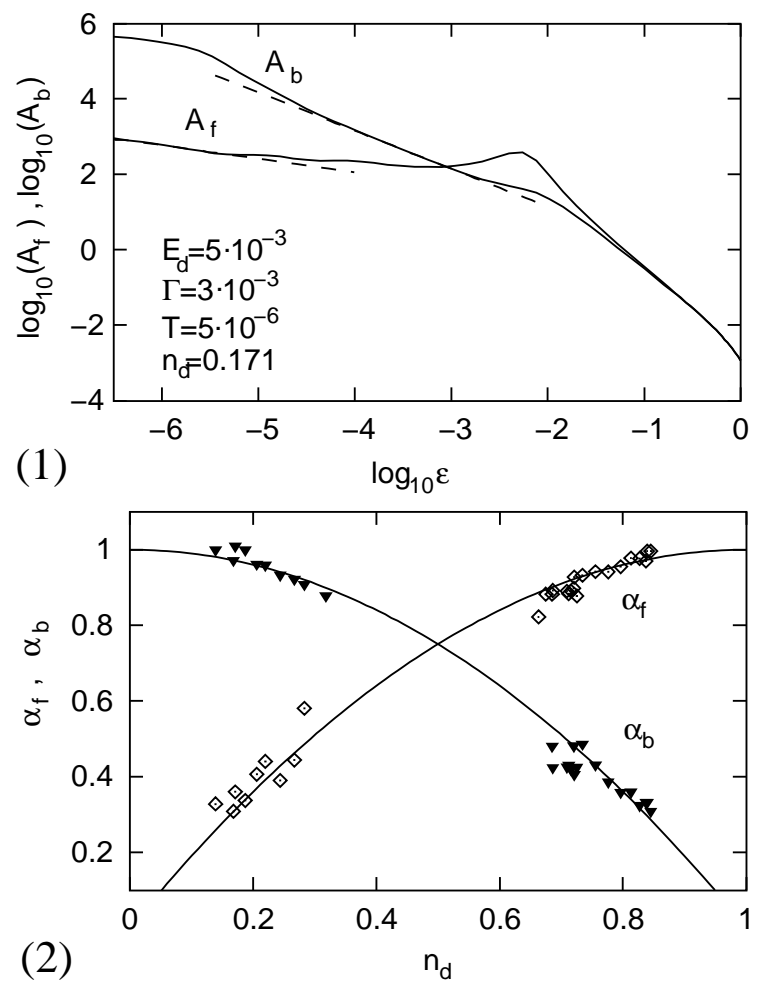

FIG. 8. (1) Pseudofermion and slave boson spectral functions $A_{f}$ and $A_{b}$ for the X-ray problem $(N=1, M=1$; $E_{d}=0.005, \Gamma=0.003$ in units of the half-bandwidth $D$ ) [48]. The slopes of the dashed lines indicate the exact threshold exponents as derived in section III B. (2) CTMA results (symbols) for the threshold exponents $\alpha_{f}$ and $\alpha_{b}$ of the spectral functions $A_{f}$ and $A_{b},(N=1, M=1)$. Solid lines: exact values (section III B).

In the multi-channel case $(N \geq 2, M \geq N)$ NCA has been shown 45 to reproduce asymptotically the correct threshold exponents, $\alpha_{f}=M /(M+N), \alpha_{b}=$ $N /(M+N)$, in the Kondo limit. Calculating the Tmatrices using NCA Green's functions (as discussed in the single-channel case) we find again a pole in the singlet channel of $T^{(c f)}$. However, in this case the CTMA does not renormalize the NCA exponents in the Kondo limit of the two-channel model, i.e. the threshold exponents obtained from the CTMA solutions are very close to the exact ones, $\alpha_{f}=1 / 2, \alpha_{b}=1 / 2$, as shown in Fig. 7 (1) b).

We note in passing that we have also solved the spinless case $(N=1, M=1)$ using the auxiliary particle method 48 . The Anderson impurity model, Eqs. (6)-(8), then reduces to a noninteracting resonant level model. In this sense, the spinless case may be seen as the most extreme case of a quantum impurity model with a Fermi liquid ground state. It does, however, remain non-trivial in the slave particle representation Eq. (11) and, therefore, constitutes a test case for the description of Fermi liquid behavior within the auxiliary particle method. As discussed in section III B, the auxiliary particle spectral functions $A_{f}, A_{b}$ in general display an infrared threshold with power law behavior which is induced by an orthogonality catastrophy analogous to that of the Xray problem. In the spinless case, $A_{f}$ and $A_{b}$ have been shown to correspond precisely to the photoemission and X-ray absorption spectral densities of the X-ray problem, respectively 48 . The results of the CTMA for the $N=1, M=1$ case are shown in Fig. 8. Good quantitative agreement of the X-ray exponents extracted from the numerical evaluation of the CTMA equations is found in the regions $n_{d} \lesssim 1$ and $n_{d} \gtrsim 0$ which may be identified with the regions of a strong (scattering phase shift $\eta_{\sigma} \simeq \pi / 2$ ) and a weak potential (scattering phase shift $\eta_{\sigma} \simeq 0$ ) scatterer.

The agreement of the CTMA exponents with their exact values in the Kondo, mixed valence and empty impurity regimes of the Spin $1 / 2$ single-channel model, in the spinless model and in the Kondo regime of the twochannel model may be taken as evidence that the $\mathrm{T}-$ matrix approximation correctly describes both the Fermi liquid $(N=1,2, M=1)$ and the non-Fermi liquid $(N=2, M=1,2)$ regimes of the $\mathrm{SU}(\mathrm{N}) \times \mathrm{SU}(\mathrm{M})$ Anderson model. Therefore, we expect the CTMA to correctly describe physically observable quantities of the $\mathrm{SU}(\mathrm{N}) \times \mathrm{SU}(\mathrm{M})$ Anderson impurity model as well.

\section{Results for physical quantities: Spin susceptibility}

We have calculated the static spin susceptibility $\chi$ of the Anderson model in the Kondo regime by solving the CTMA equations in a finite magnetic field $H$ coupled to the impurity spin and taking the derivative of the magnetization $M=\frac{1}{2} g \mu_{B}\left\langle n_{f \uparrow}-n_{f \downarrow}\right\rangle$ with respect to $H$. The resulting $\chi(T)=(\partial M / \partial H)_{T}$ is shown in Fig. 9 both for the single-channel case $(N=2, M=1)$ and for the two-channel Anderson Model $(N=2, M=2)$. It is seen that in the single-channel case CTMA correctly re- 
produces the constant Pauli susceptibility (Fermi liquid behavior) below $T_{K}$, while NCA gives an incorrect, nonanalytic temperature dependence of $\chi(T)-\chi(0) \propto-T^{1 / 3}$ at low $T$. In the two-channel case CTMA describes the non-Fermi liquid behavior, i.e. the the exact 15 logarithmic temperature dependence of the susceptibility below the Kondo scale $T_{K}$. In contrast, the NCA solution recovers the logarithmic behavior only far below $T_{K}$. Other physical quantities have been calculated for the Anderson model at low temperatures as well and will be published in forthcoming work 49 .
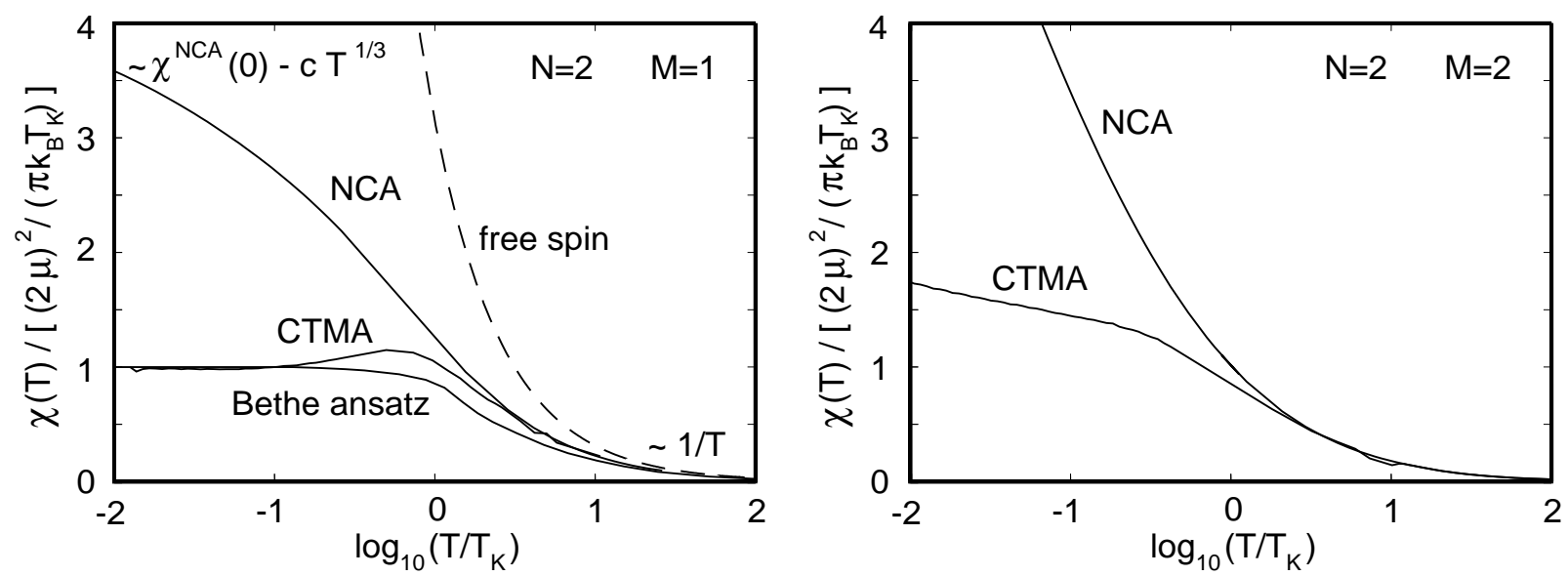

FIG. 9. Static susceptibility of the single-channel $(N=2, M=1)$ and the two-channel $(N=2, M=2)$ Anderson impurity model in the Kondo regime $\left(E_{d}=-0.8 D, \Gamma=0.1 D\right.$, Landé factor $\left.g=2\right)$. In the single-channel case, the CTMA and NCA results are compared with the Bethe ansatz result for the Kondo model [14], where the CTMA as well as the Bethe ansatz curves are scaled by their respective Kondo temperatures [14]. The CTMA susceptibility is universal in that it depends only on the ratio $T / T_{K}$ for various values of the parameters of the Anderson model (not shown).

\section{ANDERSON MODEL AT FINITE U: GENERALIZED NCA AND CTMA}

The single channel Anderson model at finite U may be represented in terms of the pseudofermion operators $f_{\sigma}$ and slave boson operators $a, b$ (discussed in the beginning of section III) as

$$
\begin{aligned}
H & =H_{c}+E_{d}\left(\Sigma_{\sigma} f_{\sigma}^{+} f_{\sigma}+2 a^{+} a\right)+U a^{+} a \\
& +V \Sigma_{\vec{k} \sigma}\left[c_{\vec{k} \sigma}^{+}\left(b^{+} f_{\sigma}+\eta_{\sigma} f_{-\sigma}^{+} a\right)+\text { h.c. }\right]
\end{aligned}
$$

subject to the constraint

$$
\sum_{\sigma} f_{\sigma}^{+} f_{\sigma}+a^{+} a+b^{+} b=1
$$

There are now two bosons, a "light" boson b (as in the case $U \rightarrow \infty$ considered before) and a "heavy" boson a. As seen from Eq. (92), the strong two-particle interaction $U$ is transformed into a potential term by this representation. The corresponding diagrams are made of propagator lines for the light boson (wiggly line, as before) and for the heavy boson (zig-zag line) as well as a new vertex, where incoming conduction electron lines and pseudofermion lines merge into an outgoing heavy boson line.

\section{A. Generating functional}

It seems straightforward to define a generalization of NCA for finite $U$ by adding to the second order skeleton diagram for the generating functional $\Phi$ a second one where the light boson is replaced by the heavy boson (see Fig. 10 a)). This approximation has been considered sometime ago 5,51 , where it was found to fail badly: Not even the Kondo energy scale is recovered in the so defined approximation. The reason for this failure is obvious: In the Kondo regime $\left(n_{d} \sim 1\right)$ the local spin is coupled to the conduction electron spin density at the impurity through the antiferromagnetic exchange coupling $J=V^{2}\left(-\frac{1}{E_{d}}+\frac{1}{E_{d}+U}\right)$. The two terms on the r.h.s. of this relation arise from virtual transitions into the empty and doubly occupied local level, which contribute equally in the symmetric case $\left|E_{d}\right|=E_{d}+U$. The symmetric occurrence of these two virtual processes in all intermediate states is not included in the simple extension of NCA proposed above. The self-energy insertions in each of the two diagrams contain always only one of the processes, leading to an effective $J$ which is only one half of the correct value. Correspondingly, the Kondo temperature $T_{K} \sim \exp (-1 / \mathcal{N}(0) J)$ comes out to scale as the square of the correct value, which is orders of magnitude too small. 


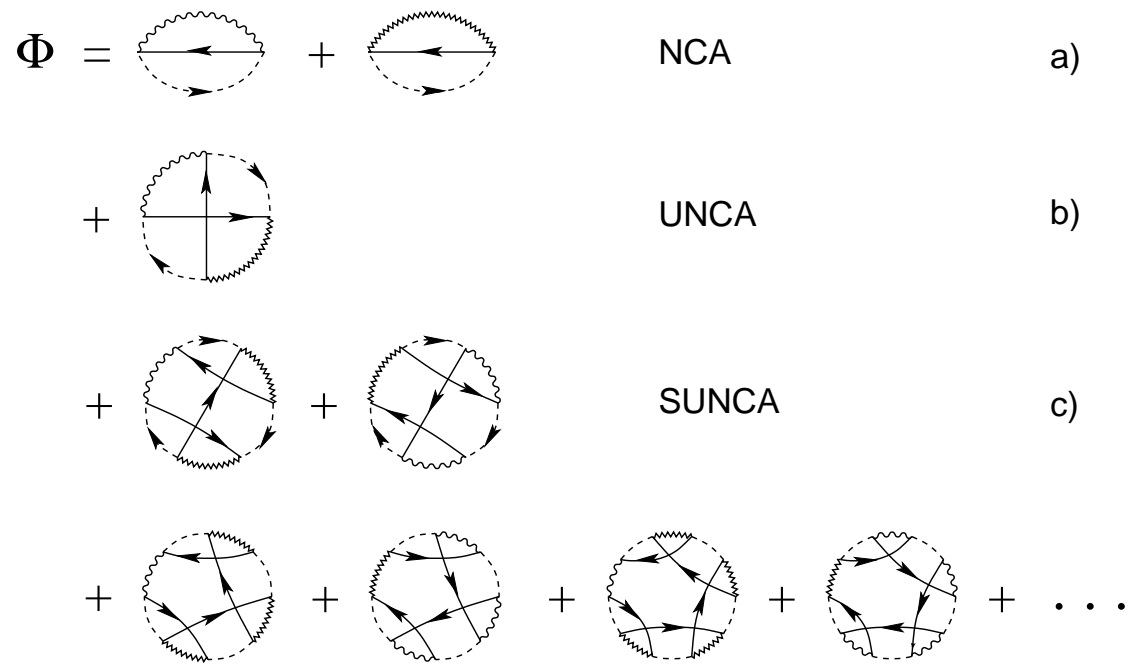

FIG. 10. Diagrammatic representation of the generating functional for the Anderson impurity model at finite $U$. Solid, dashed, wiggly and zig-zag lines correspond to conduction electron $c$, pseudofermion $f$, "light" boson $b$, and "heavy" boson $a$ propagators. a) NCA including light (empty impurity) and heavy (doubly occupied impurity) boson lines. b) UNCA containing, in addition to an infinite number of (bare) hybridization processes into the empty impurity state (non-skeleton diagrams), a single hybridization into the doubly occupied impurity state. c) Symmetrized, finite- $U$ NCA (SUNCA): The infinite series of all diagrams of a)-c) generates an approximation where the hybridization processes into the empty and doubly occupied state are treated in a symmetric way, i.e. for each contribution with a bare light boson line there is a corresponding contribution with the bare light boson line replaced by a bare heavy boson line, and vice versa.

To correct this deficiency it is necessary to include additional diagrams, restoring the symmetry between the two virtual processes, excitation into the empty or into the doubly occupied impurity state. Due to the exact projection onto the physical Hilbert space, each diagram contributing to $\Phi$ contains exactly one auxiliary particle ring (see, e.g. Fig. 5). As a first step one may add the next order diagram to $\Phi$ (see Fig. 10 b)). As will be seen below, this approximation, later referred to as UNCA, helps to recover a large part of the correct behavior of $T_{K}$. A completely symmetric treatment, however, requires the summation of infinite classes of diagrams. These diagrams are generated by replacing a light boson line with a heavy boson line in each of the bare (nonskeleton) diagrams of the elementary NCA diagrams of Fig. 11. Each replacement leads to a crossing of conduction electron lines spanning one fermion and at most two boson lines. As a result, the class of diagrams of $\Phi$ shown in Fig. 10 c) are obtained. These diagrams look similar to the CTMA diagrams shown in Fig. 5 , but contain one light boson line and an arbitrary number of heavy boson lines, or vice versa. Diagrams with, for example, two light boson lines and an arbitrary number of heavy boson lines (and conduction electron lines spanning at most one fermion line) are reducible and do not appear. We will call the approximation defined by the generating functional given by the sum of the diagrams of Figs. 10 a)c) "symmetrized, finite- $U$ non-crossing approximation" (SUNCA) 253. It is interesting to note that the sym- metric treatment of light and heavy bosons leads to diagrams for $\Phi$ of a structure similar to the ones defining the CTMA (Fig. 5). The above approximation corresponding to the CTMA at $U \rightarrow \infty$, termed "symmetrized, finite- $U$ Conserving T-matrix Approximation (SUCTMA) is thus defined in a natural way by summing up all skeleton diagrams with an arbitrary number of light or heavy boson lines, dressed by conduction electron lines spanning only one fermion line. The rational for keeping only these crossings is that (as explained in the appendix),

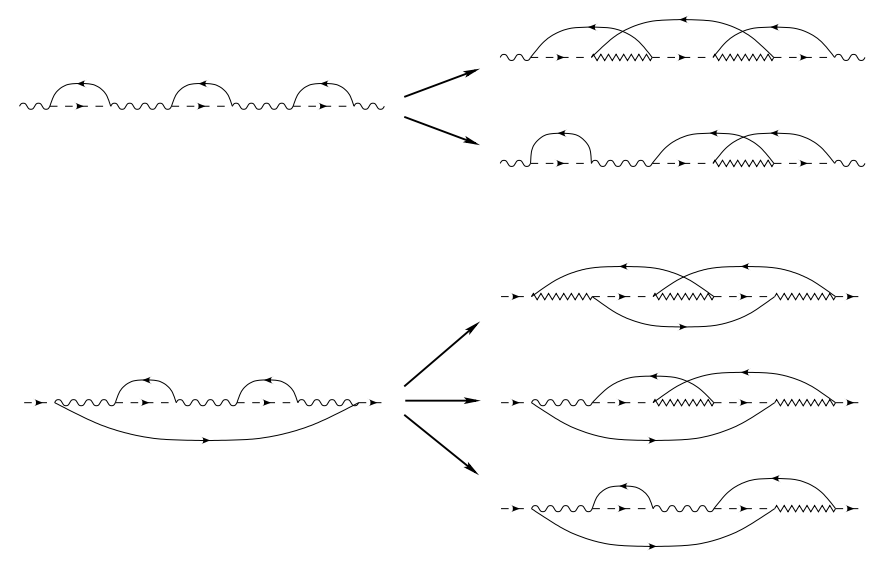

FIG. 11. Two examples of how diagrams involving hybridization into the doubly occupied impurity state are generated from the bare noncrossing diagrams of the infinite $U$ case by replacing light with heavy boson lines (see text). 
diagrams containing conduction electron lines spanning two or more fermion lines may be grouped into sets where the most singular contributions cancel. Thus, the SUCTMA is defined by adding to the diagrams of the
SUNCA the CTMA diagrams (Fig. 5) with (only) light boson lines or (only) heavy boson lines. The SUCTMA equations have not yet been evaluated.
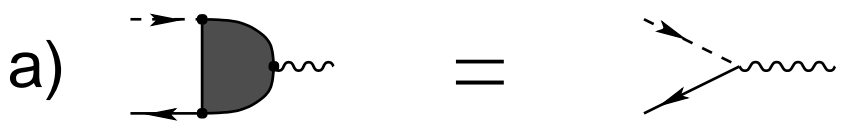
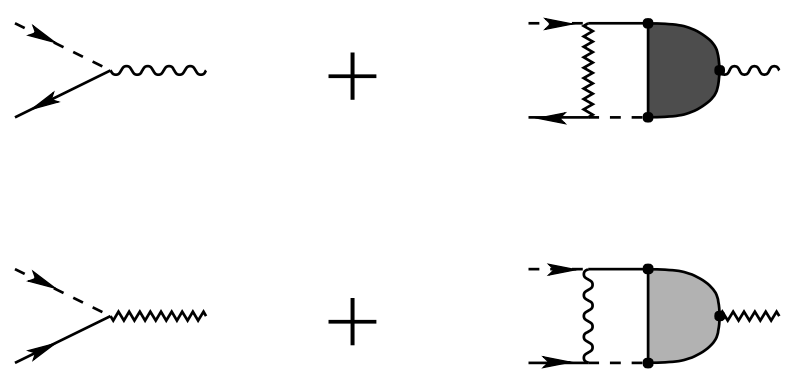

FIG. 12. Diagrammatic representation of the Bethe-Salpeter equations for a) the renormalized light boson (empty impurity) and b) the renormalized heavy boson (doubly occupied impurity) vertex, as generated by the SUNCA Luttinger-Ward functional (Fig. 11).

\section{B. Results of SUNCA}

The pseudoparticle self-energies $\Sigma_{a}, \Sigma_{b}$ and $\Sigma_{f}$ as well as the local conduction electron self-energy $\Sigma_{c}$ are obtained as funcional derivatives with respect to the corresponding Green's functions. They can be expressed in terms of the two vertex functions defined in Fig. 12 as solutions of Bethe-Salpeter equations. It is seen that the vertex functions are obtained by ladder summations involving a heavy boson or a light boson line as the rung of the ladder. These three-point vertex functions depend on two frequencies and are much less difficult to calculate than the four-point T-matrices of the CTMA. The full expressions of the self-energies are given in Fig.13. The local d-electron Green's function after projection is again proportional to $\Sigma_{c}$ :

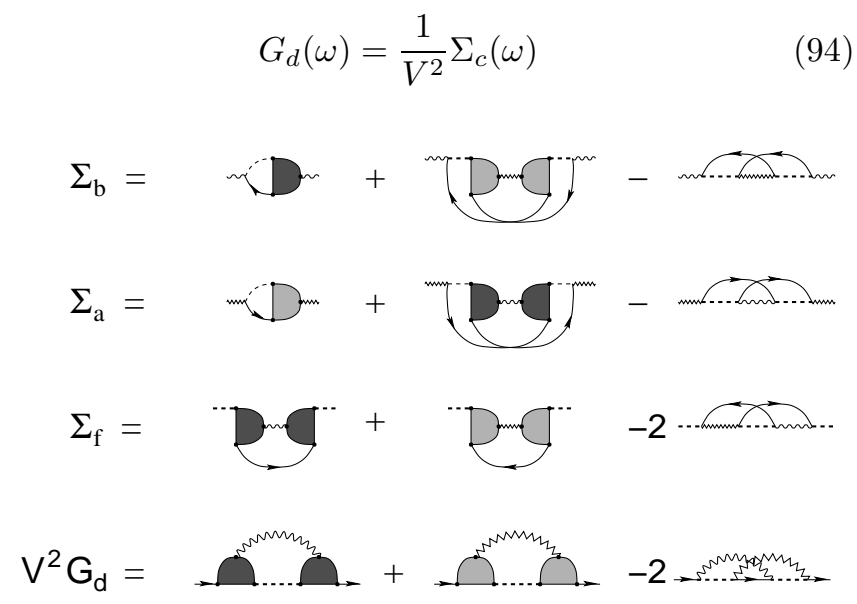

FIG. 13. Diagrammatic representation of the auxiliary particle selfenergies of SUNCA in terms of the renormalized hybridization vertices, defined in Fig. 12. Note that in each line the third diagram is subtracted in order to avoid double counting of terms within the first two diagrams.
The self-consistent set of equations defined by Fig. 13 and the expression for the pseudo-particle Green's function, Eq. (21), supplemented by the expression for the heavy boson Green's function

$$
\mathcal{G}_{a}\left(i \omega_{n}\right)=\left\{i \omega_{n}-\left(2 E_{d}+U\right)-\Sigma_{a}\left(i \omega_{n}\right)\right\}^{-1}
$$

have been solved numerically 52,53 . As expected, the pseudoparticle spectral functions are found to display power law divergencies at the infrared threshold. The exact power law exponents, derived from the Friedel sum rule argument based on the assumed Fermi liquid ground state (section III B), are given by

$$
\begin{aligned}
& \alpha_{f}=n_{d}-\frac{n_{d}^{2}}{2} \\
& \alpha_{b}=1-\frac{n_{d}^{2}}{2} \\
& \alpha_{a}=-1+2 n_{d}-\frac{n_{d}^{2}}{2}
\end{aligned}
$$

The threshold exponents obtained from the SUNCA solution are shown in Fig. 14 as functions of the average impurity occupation number $n_{d}$. They agree with the exact results in the Kondo limit $\left(n_{d} \rightarrow 1\right)$ where $\alpha_{f}=\alpha_{b}=\alpha_{a}=\frac{1}{2}$, but deviate for $n_{d}<1$. It is expected that the SUCTMA will recover the correct exponents, as does the CTMA in the case of infinite $U$. The d-electron spectral function for the symmetric Anderson model as obtained in SUNCA is shown in Fig. 15 (left panel). In this case the Kondo resonance is located exactly at $\omega=0$. Its width is a measure of the Kondo temperature $T_{K}$. The three curves shown correspond to the elementary NCA (Fig. 10 a)), the UNCA (Fig. 10 b)) and the SUNCA. The inset shows that for the parameters chosen the $T_{K}$ obtained in NCA is too small by a 


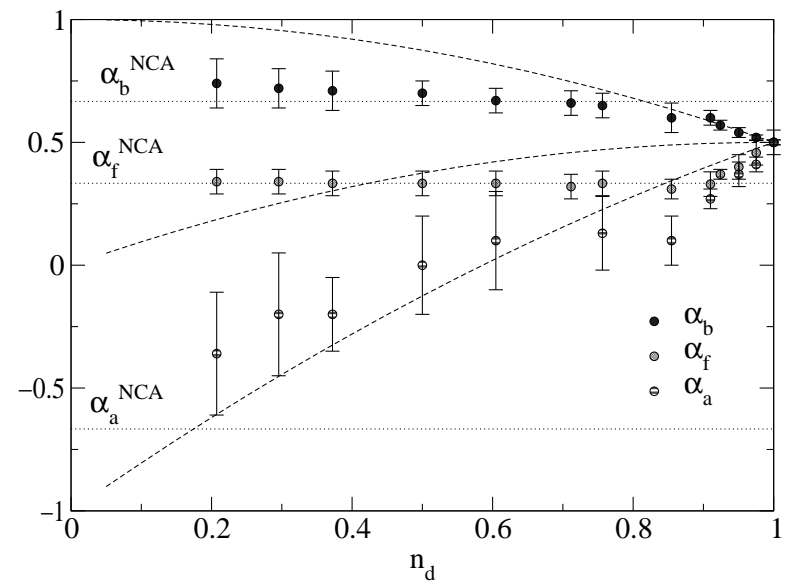

FIG. 14. Infrared threshold exponents of the auxiliary particle spectral functions for the case of finite $U$ in dependence of the the impurity occupation $n_{d}$. Dashed curved lines: exact results (Eqs. (96)-(98)); horizontal lines: NCA results; data points with error bars: SUNCA results. In the Kondo limit $\left(n_{d} \rightarrow 1\right)$ the exact exponents are recovered, while in the mixed valence and empty impurity regime the SUNCA results for $\alpha_{f}$ and $\alpha_{b}$ cross over to the NCA values.

factor $10^{-2}$, whereas the UNCA is only a factor of $1 / 3$ off. Contrary to Ref.50 we do not find that the UNCA is sufficient to recover $T_{K}$. In Fig. 15 (right panel) the results of the SUNCA for $T_{K}$ as determined from the width of the Kondo resonance are compared to the exact result,

$$
T_{K}=\min \left\{\frac{1}{2 \pi} U \sqrt{I}, \sqrt{D \Gamma}\right\} \mathrm{e}^{(-\pi / I)},
$$

where $I=\frac{2 \Gamma U}{\left|E_{d}\right|\left(U+E_{d}\right)}$. The agreement is seen to be very good for a large range of parameters $I$ and $E_{d}$.

\section{CONCLUSION}

We have reviewed a technique to describe correlated quantum impurity systems with strong onsite repulsion, which is based on a conserving formulation of the auxiliary boson method. The conserving scheme allows to implement the conservation of the local charge $Q$ without taking into account time dependent fluctuations of the gauge field $\lambda$, while exactly projecting the quantum dynamics onto the physical subspace of no double occupancy of sites (in the limit of infinitely strong on-site repulsion). Any spurious condensation of the auxiliary boson field is avoided in this way.

By including the physically dominant contributions, spin flip processes in the Kondo regime, and spin as well as charge fluctuation processes in the mixed valence and empty impurity regimes, the method recovers the Fermi liquid ground state of the single-channel Anderson impurity model as well as the non-Fermi-liquid low-temperature behavior of the two-channel Anderson model: The correct infrared threshold exponents of the auxiliary particle propagators, which are identified as indicators for Fermi or non-Fermi liquid behavior, are obtained in both cases. Physical quantities, like the magnetic susceptibility, are correctly described both in the Fermi and in the non-Fermi liquid cases of the model over the complete temperature range, including the crossover to the correlated many-body state at the lowest temperatures, which has previously been notoriously difficult to obtain within a diagrammatic many-body theory.
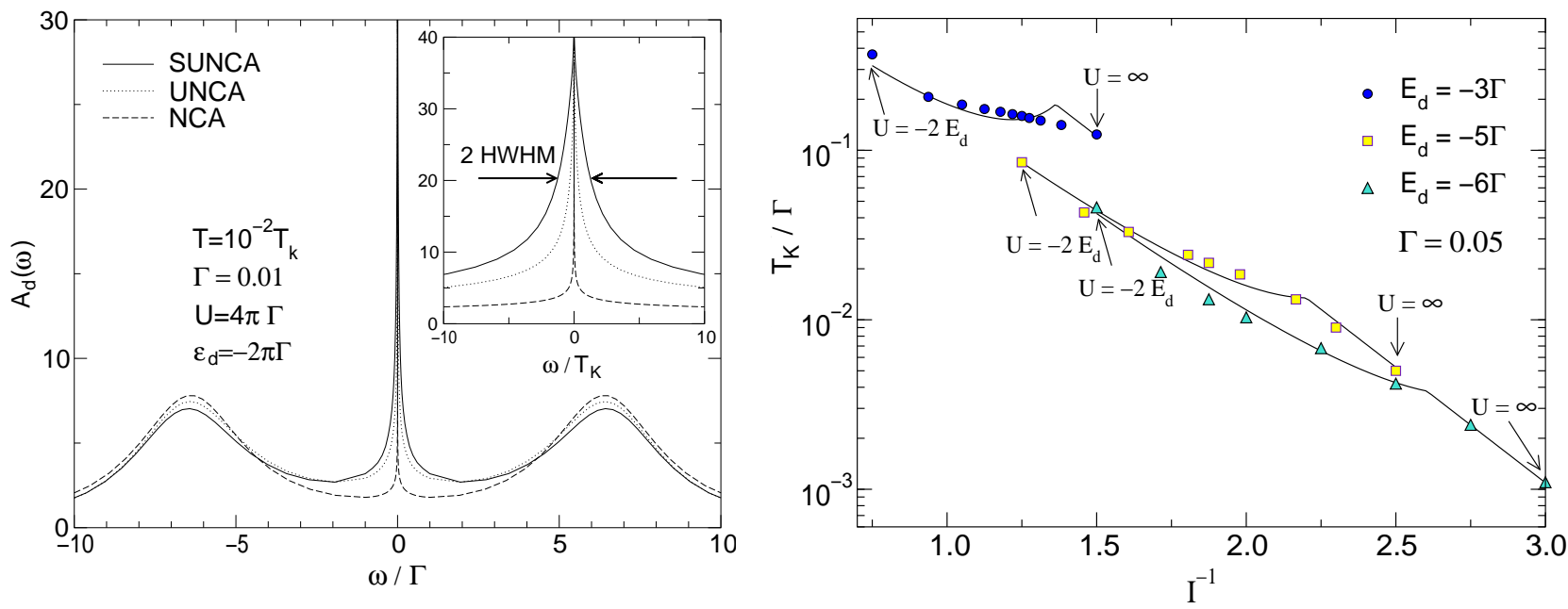

FIG. 15. Left panel: Local electron spectral function calculated using NCA, UNCA, and SUNCA. The Kondo temperature is determined by the peak width. It is seen that in NCA it comes out orders of magnitude too low. - Right panel: Kondo Temperature for various parameters $E_{d}, U$ and fixed $\Gamma$. Solid lines represent the results from the Bethe ansatz solutions, Eq. (99). Data points are the SUNCA results determined from the width of the Kondo peak in the d-electron spectral function. 
We have also presented a generalization of the auxiliary particle method for finite on-site repulsion $U$ and solved the corresponding equations on the level of a generalized non-crossing approximation (SUNCA), where virtual excitations into the empty or into the doubly occupied impurity state are treated in a fully symmetric way. In this way the correct low-temperature scale of the finite-U Anderson model was obtained. The controlled treatment of a finite on-site repulsion is a precondition for applying the auxiliary particle method to models of the Hubbard type or - within the scheme of the dynamical mean field theory (limit of infinite dimensions) - of the Anderson impurity type, where a Mott-Hubbard metal-insulator transition occurs as a function of band filling for finite $U$. As a standard diagram technique the conserving auxiliary particle method has the potential to be applicable to problems of correlated systems on a lattice as well as to mesoscopic systems out of equilibrium via the Keldysh technique. These developments are currently under investigation.

Collaboration and discussions with S. Böcker, T. A. Costi, K. Haule, S. Kirchner, and Th. Schauerte are gratefully acknowledged. This work is supported in part by DFG through SFB 195 and by a grant of computer time of the High-Performance Computing Center Stuttgart.

\section{APPENDIX: INFRARED CANCELLATION OF NON-CTMA DIAGRAMS}

The CTMA is not only justified on physical grounds by the inclusion of the maximum number of spin flip and charge fluctuation processes at any given order of perturbation theory, but also by an infrared cancellation of all diagrams not included in the CTMA. In the following we will prove this cancellation theorem.
(1) Power counting. - Each auxiliary particle loop carries a factor of the fugacity $\exp (-\beta \lambda)$, which vanishes upon projection onto the $Q=1$ subspace, $\lambda \rightarrow \infty$. Therefore, an arbitrary $f$ or $b$ self-energy diagram consists of one single line of alternating fermion and boson propagators, with the hybridization vertices connected by conduction electron lines in any possible way, as shown in Fig. 16 (see also 40 ). Such a fermion self-energy skeleton diagram of loop order $L$ is calculated as

$$
\begin{aligned}
\Sigma_{f}^{(L)}(\omega) & =(-1)^{3 L-1+L_{s p}} N^{L_{s p}} M^{L_{c h}} \Gamma^{L} \int \frac{d \varepsilon_{1}}{\pi} \ldots \frac{d \varepsilon_{L}}{\pi} f\left(\varepsilon_{1}\right) \ldots f\left(\varepsilon_{L}\right) A_{c}^{0}\left(s_{1} \varepsilon_{1}\right) \ldots A_{c}^{0}\left(s_{L} \varepsilon_{L}\right) \\
& \times G_{b}\left(\omega+\omega_{1}\right) G_{f}\left(\omega+\omega_{1}+\omega_{1}^{\prime}\right) \ldots G_{b}\left(\omega+\sum_{i=1}^{k} \omega_{i}+\sum_{i=1}^{k-1} \omega_{i}^{\prime}\right) G_{f}\left(\omega+\sum_{i=1}^{k}\left(\omega_{i}+\omega_{i}^{\prime}\right)\right) \ldots G_{b}\left(\omega+\omega_{L}\right)
\end{aligned}
$$

where $G_{f, b}$ are the renormalized, i.e. power law divergent auxiliary particle propagators, and $L_{s p}$ and $L_{c h}$ denote the number of spin (or fermion, $c-f$ ) loops and the number of channel (or $c-b$ ) loops contained in the diagram, respectively. Spin and channel indices are not shown for simplicity. Each of the auxiliary particle frequencies $\omega_{i}, \omega_{i}^{\prime}$ coincides with one of the integration variables $\varepsilon_{j}$, $j=1, \ldots, L$, in such a way that energy is conserved at each hybridization vertex. This implies that the sign of the frequency carried by a $c$-electron line is $s_{i}=+$, if the $c$-electron line runs from right to left, and $s_{i}=-$, if it runs from left to right in Fig. 16. An analogous expression holds for the slave boson self-energy diagrams.

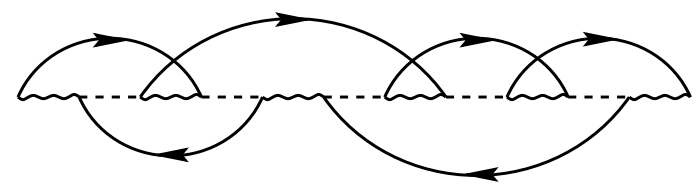

FIG. 16. Typical pseudofermion self-energy skeleton diagram of loop order $L=6$, containing $L_{s p}=1$ spin (or fermion) loop and $L_{c h}=1$ channel loop.
By substituting $x_{j}=\varepsilon_{j} / \omega, j=1, \ldots, L$ and factoring out $\omega^{-\alpha_{f,(b)}}$ from each fermion (boson) propagator, the infrared behavior of the term Eq. (A1) is deduced as

$$
\operatorname{Im} \Sigma_{f, b}^{(L)}(\omega)=C \omega^{\alpha_{f, b}+L\left(1-\alpha_{f}-\alpha_{b}\right)}
$$

where $C$ is a finite constant. Clearly, when the NCA solutions are inserted for the propagators $G_{f, b}$, i.e. $\alpha_{f}+\alpha_{b}=$ 1 , their power law behavior is just reproduced by any term of the form Eq. A1. However, this is no longer the case for the exact propagators in the Fermi liquid regime $(M<N)$, where in general $\alpha_{f}+\alpha_{b}>1$. Thus, the infinite resummation of terms to arbitrary loop order is unavoidable in this case.

(2) Infrared cancellation.- As discussed in section VI $\mathrm{B}$, the CTMA is equivalent to the self-consistent summation of all skeleton free energy diagrams, where a conduction electron line spans at most two hybridization vertices (Fig. 5). Thus, any skeleton self-energy diagram not included in CTMA contains at least one conduction electron "arch" which spans four (or more) vertices, with 


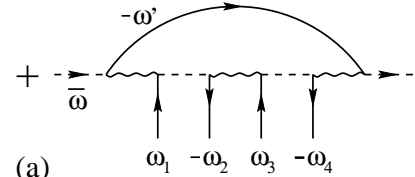

(a)
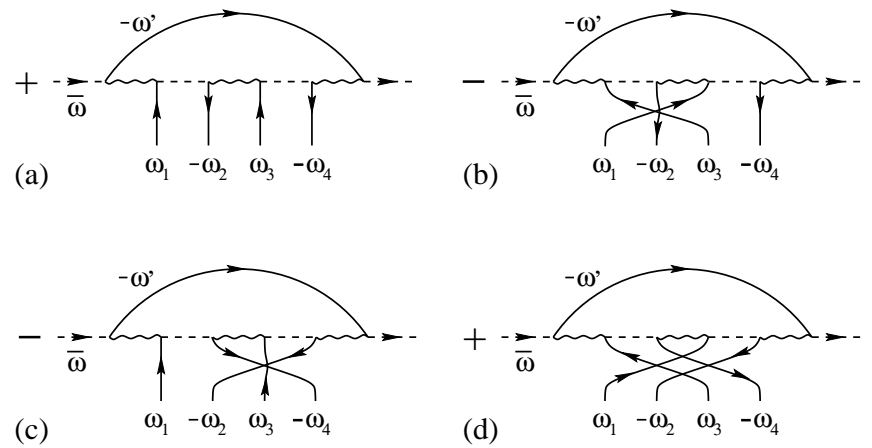

FIG. 17. Set of contributions to skeleton diagrams not contained in CTMA which cancel in the infrared limit to leading and subleading order in the external frequency, $\omega \rightarrow 0$. four conduction lines reaching from inside to outside of the arch as shown in Fig. 17(a). For each such diagram there exists another skeleton, which differs from Fig. 17(a) only in that the end points of two conduction lines inside the arch are interchanged (Fig. 17(b)). The corresponding permutation of fermionic operators implies a relative sign between the terms Fig. 17(a) and (b). Without loss of generality we now assume $\omega>0$ for the external frequency of the self-energy. The leading infrared singular behavior of the term (A1) arises from those parts of the integrations, where the arguments of the $G_{f}, G_{b}$ are such that the divergences of all propagators lie within the integration range. This implies at least $-\omega \leq \varepsilon_{j} \leq 0, j=1, \ldots, L$. Therefore, the terms corresponding to Fig. 17(a), (b) differ only in the frequency arguments of the Green's functions inside the arch, and at temperature $T=0$ the leading infrared behavior of their sum reads,

$$
\begin{aligned}
& \Sigma_{f}^{(L, a)}(\omega)+\Sigma_{f}^{(L, b)}(\omega)^{\omega \rightarrow 0}=(-1)^{3 L-1+L_{s p}} N^{L_{s p}} M^{L_{c h}} \Gamma^{L} \times \\
& \int_{-\omega}^{0} \frac{d \varepsilon_{1}}{\pi} \ldots \frac{d \varepsilon_{L}}{\pi} F\left(\omega,\left\{\varepsilon_{j}\right\}\right)\left[G_{f}\left(\bar{\omega}+\omega^{\prime}+\omega_{1}\right) G_{b}\left(\bar{\omega}+\omega^{\prime}+\omega_{1}+\omega_{2}\right)-G_{f}\left(\bar{\omega}+\omega^{\prime}+\omega_{3}\right) G_{b}\left(\bar{\omega}+\omega^{\prime}+\omega_{3}+\omega_{2}\right)\right]
\end{aligned}
$$

Here $\bar{\omega}$ denotes the sum of all frequencies $\omega, \varepsilon_{j}$ entering the diagrammatic part, Fig. 17, from the left, and $F\left(\omega,\left\{\varepsilon_{j}\right\}\right)$ consists of all terms which are not altered by interchanging the $c$-electron lines. In the infrared limit, $\omega_{1}-\omega_{3} \rightarrow 0$, the term in square brackets may be written as

$$
\frac{d}{d \bar{\omega}}\left[G_{f}\left(\bar{\omega}+\omega^{\prime}\right) G_{b}\left(\bar{\omega}+\omega^{\prime}+\omega_{2}\right)\right]\left(\omega_{1}-\omega_{3}\right)
$$

and upon performing the integrations over $\omega_{1}, \omega_{3}$ the difference $\left(\omega_{1}-\omega_{3}\right)$ leads to an additional factor of $\omega$. A similar cancellation of the leading infrared singularity occurs between the terms shown in Fig. 17(c), (d). In an analogous way it may be shown that combining the terms Fig. 17 (a)-(d) leads to a factor of $\omega^{2}$ compared

${ }^{1}$ P.A. Lee, T.M. Rice, J.W. Serene, L.J. Sham, and J.W. Wilkins, Comments on Condensed Matter Physics 12, 99 (1986).

2 P.W. Anderson, Science 235, 1196 (1987); and "The Theory of Superconductivity in the High-T $c$ Cuprates", (Princeton University Press, 1997).

${ }^{3}$ For a review see: "Perspectives in Quantum Hall Effects", eds. S. Das Sarma and A. Pinczuk (Wiley, New York, 1996).

${ }^{4}$ A.A. Abrikosov, Physics 2, 21 (1965). to the power counting result for one single term. Thus, the infrared singularity of all non-CTMA terms of loop order $L$ is weaker than the $L$ th order CTMA terms by at least $O\left(\omega^{2}\right)$,

$$
\Sigma_{f, b}^{(L, a)}(\omega)+\ldots+\Sigma_{f}^{(L, d)}(\omega) \stackrel{\omega}{\vec{\propto}^{0}} \omega^{\alpha_{f, b}+L\left(1-\alpha_{f}-\alpha_{b}\right)+2} .
$$

It should be emphasized that in the above derivation, $L$ appears only as a parameter and, thus, the cancellation theorem holds for arbitrarily high loop order $L$. This proves that the CTMA captures the leading and subleading infrared singularities $(\omega \rightarrow 0)$ at any given order $L$.

${ }^{5}$ S.E. Barnes, J. Phys. F 6, 1375 (1976); 7, 2637 (1977).

${ }^{6}$ P. Coleman, Phys. Rev. B 29, 2 (1984).

${ }^{7}$ D.M. Newns, N. Read, J. Phys. C 16, 3273 (1983); Adv. Phys. 36, 799 (1988).

8 A.C. Hewson, "The Kondo Problem to Heavy Fermions" (Cambridge University Press, 1993).

${ }^{9}$ D.L. Cox and A. Zawadowski, Adv. Phys. 47, 599 (1998).

${ }^{10}$ See, e.g., related articles in Mesoscopic Electron Transport, L. L. Sohn, L. P. Kouwenhoven and G. Schön eds., NATO ASI Series E: Applied Sciences 345 (Kluwer Science Publishers, Dordrecht, Boston, London, 1997).

${ }^{11}$ P.W. Anderson, Phys. Rev. 124, 41 (1961).

12 J.R. Schrieffer and P.A. Wolff, Phys. Rev. 149, 491 (1966). 
${ }^{13}$ K.G. Wilson, Rev. Mod. Phys. 47, 773 (1975).

${ }^{14}$ N. Andrei, K. Furuya and J.H. Löwenstein, Rev. Mod. Phys. 55, 331 (1983).

${ }^{15}$ P.B. Wiegmann and A.M. Tsvelik, J. Phys. C 12, 2281 and 2321 (1983).

${ }^{16}$ P. Noziéres, J. Low Temp. Phys. 17, 31 (1974).

${ }^{17}$ N. Andrei and C. Destri, Phys. Rev. Lett. 52, 364 (1984).

18 A.M. Tsvelik, J. Phys.C 18, 159 (1985).

${ }^{19}$ I. Affleck and A.W.W. Ludwig, Nucl. Phys. 352, 849, (1991); B360, 641, (1991); Phys. Rev. B 48, 7297 (1993).

${ }^{20}$ K. Yamada, Prog. Theor. Phys. 53, 970 (1975); ibid. 54, 316 (1975); ibid. 55, 1345 (1976); K. Yosida and K. Yamada, Prog. Theor. Phys. 53, 1286 (1975).

${ }^{21}$ D. L. Cox, N. E. Bickers and J. W. Wilkins, Phys. Rev. B 36, 2036 (1987).

${ }^{22}$ This means that the auxiliary particle propagators are not calculated in the canonical $(Q=1)$ ensemble. The projection onto the $Q=1$ sector of Fock space is achieved only when they are combined to calculate expectation values of physically observable operators like $G_{d \sigma},\langle\vec{S}\rangle$ etc. The latter can be seen explicitly, e.g., from Eq. (54), 2nd equality.

${ }^{23}$ P. W. Anderson, Phys. Rev. Lett. 18, 1049 (1967).

${ }^{24}$ P. Nozières and C. T. De Dominicis, Phys. Rev. 178, 1073; 1084; 1097 (1969).

${ }^{25}$ G. D. Mahan, Many-Particle Physics, 2nd ed., pp. 732 (Plenum Press, New York, 1990) gives an overview.

${ }^{26}$ K. D. Schotte and U. Schotte, Phys. Rev. 185, 509 (1969).

${ }^{27}$ B. Menge and E. Müller-Hartmann, Z. Phys. B73, 225 (1988).

28 T.A. Costi, P. Schmitteckert, J. Kroha and P. Wölfle, Phys. Rev. Lett. 73, 1275 (1994).

29 T.A. Costi, P. Schmitteckert, J. Kroha and P. Wölfle, Physica (Amsterdam) 235-240C, 2287 (1994).

${ }^{30}$ S. Fujimoto, N. Kawakami and S.K. Yang, J.Phys.Korea 29, S136 (1996).

${ }^{31}$ D. M. Newns and N. Read, J. Phys. C16, 3273 (1983); Adv. Phys. 36, 799 (1988).

32 S. Elitzur, Phys. Rev. D 12,3978 (1975).

${ }^{33}$ A. Jevicki, Phys. Lett. B 71, 327 (1977).
${ }^{34}$ F. David, Comm. Math. Phys. 81, 149 (1981).

${ }^{35}$ I.D. Lawrie, J. Phys. A 18, 1141 (1985).

${ }^{36}$ B. Jin and Y. Kuroda, J. Phys. Soc. Japan 57, 1687 (1988).

37 T. Matsuura et al., J. Phys. Soc. Japan 66, 1245 (1997).

${ }^{38}$ G. Sellier, Diploma Thesis, Universität Karlsruhe, unpublished (1998).

${ }^{39}$ G. Baym and L.P. Kadanoff, Phys. Rev. 124, 287 (1961); G. Baym, Phys. Rev. 1271391 (1962).

${ }^{40}$ H. Keiter and J. C. Kimball, J. Appl. Phys. 42, 1460 (1971); N. Grewe and H. Keiter, Phys. Rev B 24, 4420 (1981).

41 Y. Kuramoto, Z. Phys. B 53, 37 (1983); H. Kojima, Y. Kuramoto and M Tachiki, ibid. 54, 293 (1984); Y. Kuramoto and H. Kojima, ibid. 57, 95, (1984); Y. Kuramoto, ibid. 65, 29, (1986).

${ }^{42}$ E. Müller-Hartmann, Z. Phys. B57, 281 (1984).

${ }^{43}$ T.A. Costi, J. Kroha, and P. Wölfle, PRB 53, 1850 (1996).

${ }^{44}$ N. E. Bickers, Rev. Mod. Phys. 59, 845 (1987); N. E. Bickers, D. L. Cox \& J. W. Wilkins, Phys. Rev. B36, 2036 (1987).

45 D. L. Cox and A. E. Ruckenstein, Phys. Rev. Lett. 71, 1613 (1993).

${ }^{46}$ F. Anders and N. Grewe, Europhys. Lett. 26, 551 (1994); F. Anders, J. Phys. Cond. Mat. 7, 2801 (1995).

47 J. Kroha, P. Wölfle and T. A. Costi, Phys. Rev. Lett. 79, 261 (1997).

48 Th. Schauerte, J. Kroha, and P. Wölfle, Phys. Rev. B 62, 4394 (2000).

49 S. Kirchner, J. Kroha, and P. Wölfle, in preparation.

50 Th. Pruschke and N. Grewe, Z. Phys. B 74, 439 (1989).

${ }^{51}$ I. Holm and K. Schönhammer, Solid State Comm. 69, 10, 969 (1989).

${ }^{52}$ K. Haule, S. Kirchner, J. Kroha, and P. Wölfle, in Proceedings of the NATO Advanced Research Workshop on "Size Dependent Magnetic Scattering", Pecs, Hungary 2000 (Kluwer Academic Publishers, in press).

${ }^{53}$ K. Haule, S. Kirchner, J. Kroha, and P. Wölfle, condmat/0105490 (2001). 